\title{
Developing Grain Boundary Diagrams for Multicomponent Alloys
}

\author{
Naixie Zhou and Jian Luo* \\ Department of NanoEngineering; Program of Materials Science and Engineering, University of \\ California, San Diego, CA 92093-0448, USA
}

\begin{abstract}
:
Impurity-based, premelting-like, intergranular films (IGFs, a common type of grain boundary complexion) can form in various materials and influence sintering, creep, and microstructure development. A thermodynamic framework is presented to forecast the formation and stability of these premelting-like grain boundary complexions (a.k.a. interfacial "phases" that are thermodynamically two dimensional) in multicomponent alloys to consider the interactions of multiple alloying elements. Key thermodynamic parameters that control the interfacial segregation and disordering behaviors have been identified and systematically examined. Subsequently, ternary and quaternary grain boundary diagrams have been computed and used to forecast the sintering behaviors of W-Ni- $M(M=\mathrm{Fe}, \mathrm{Co}, \mathrm{Cr}, \mathrm{Zr}, \mathrm{Nb}$ and $\mathrm{Ti})$ and Mo-Si-B-M $(M=\mathrm{Ni}, \mathrm{Co}$ and $\mathrm{Fe})$ systems. This work supports a long-range scientific goal of extending bulk computational thermodynamics and CALPHAD methods to interfaces and developing grain boundary complexion (interfacial "phase") diagrams as extensions to bulk phase diagrams, which can be a generally useful materials science tool.
\end{abstract}

Keywords: CALPHAD; Grain boundary complexion; Interfacial Thermodynamics; Materials Genome Initiative; Premelting;

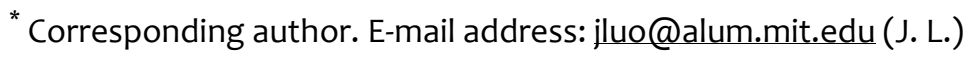




\section{Introduction}

It is now well established that the surface of ice starts to "melt" well below $0{ }^{\circ} \mathrm{C}[1-3]$. This phenomenon, known as surface melting or premelting, was also confirmed for other unary solids such as lead [1-3]. In the middle $19^{\text {th }}$ century, Faraday originally proposed the existence of premelting and used it to explain why two blocks of ice can freeze together and a snowball can hold together below $0{ }^{\circ} \mathrm{C}[1-3]$, which are analogous to the sintering phenomenon studied by materials scientists. Geophysicists also believe that grain boundary (GB) premelting, enhanced by the presence of minor impurities, can play an important role in controlling glacier motion [1-3], which is related to the Coble creep phenomenon known in the materials research community. In 1980s, materials scientists had sought to confirm the existence of GB premelting in unary metals experimentally [4]; although GB premelting was later discovered in a colloidal crystal [5], the significance and importance of GB premelting in real unary materials remain elusive. Nevertheless, in multicomponent systems, the interfacial disordering can be enhanced by a concurrent GB adsorption (a.k.a. segregation); consequently, impurity-based, premelting-like, intergranular films (IGFs) can be stabilized over at larger undercoolings and influence a broad range of materials properties and fabrication processing [6-21]. Specifically, indirect measurements of GB diffusivity and chemistry suggested the existence of GB premelting-like behaviors in $\mathrm{Cu}[6-8]$ and $\mathrm{Fe}[7$, $9,10]$ based alloys. More recently, impurity-based, premelting-like IGFs have been observed directly by high resolution transmission electron microscopy (HRTEM) in W [15, 16] and Mo [17-19] based alloys as well as several oxide systems [11-14].

Tang et al. [14] proposed that subsolidus IGFs in binary alloys form from coupled GB premelting and prewetting transitions [20] using a diffuse-interface model extended from the Cahn model [22], and this diffuse-interface model has been further elaborated by Mishin et al. using the $\mathrm{Cu}-\mathrm{Ag}$ system as an example [21]. In a broader context, GB premelting and prewetting can be considered as the representative GB structural (disordering) and chemical (adsorption) transitions, respectively, and they are often coupled in multicomponent systems [14, 21]. In 1968, Hart already proposed that GBs can be considered as 2-D interfacial phases that may undergo transformations (a.k.a. transitions) [23, 24]. Subsequent models developed by Hondros and Seah [25, 26], Cahn [22, 27-29], and many other researchers [18, 20, 21, 3042] further elaborated this concept. In ceramic materials, impurity-based IGFs can persist well above the bulk solidus lines with nanometer thicknesses limited by attractive van der Waals London dispersion interactions [14, 30, 31, 43, 44]. Clarke first proposed that such IGFs have an equilibrium thickness [30, 31] and Cannon suggested that they can equivalently be interpreted as a class of structurally-disordered multilayer adsorbates [45]. Later, Tang, Carter and Cannon [20, 32] named such "2-D interfacial phase" as "complexions" based on an argument that they are not "phases" according to the rigorous Gibbs 
definition, and related terminologies were discussed in two recent reviews [34, 46]. In 2007, Dillon and Harmer [47-50] reported the discovery of a series of six discrete $\mathrm{GB}$ complexions in $\mathrm{Al}_{2} \mathrm{O}_{3}$ based ceramics, and similar complexions have also been observed in metals [18, 19, 46, 51-54]; this series of Dillon-Harmer complexions can be considered as derivatives of IGFs (a.k.a. multilayer adsorbates) with discrete (equilibrium) thicknesses of $0,1,2,3, x$, and $\infty$ atomic layers [40, 42, 46, 55]. Moreover, the existence of first-order GB complexion transitions was experimentally evident in $\mathrm{Cu}$ [6-8], $\mathrm{Fe}$ [7, 9, 10], $\mathrm{Si}[51]$ and $\mathrm{TiO}_{2}$ [56] based systems and supported by recent atomistic modeling [57, 58]; analogous firstorder transitions have also been discovered at free surfaces [59-62].

Understanding GB complexions and transitions is both scientifically significant and technologically important. Specifically, enhanced mass transport in the impurity (sintering aid) based, premelting-like IGFs can lead to accelerated sintering below the bulk solidus lines, which explains a long-standing mystery regarding the origin of solid-state activated sintering [12, 16, 18, 42]. The discoveries of GB complexions and transitions also provided new insights into the understanding of the atomic-level mechanisms for abnormal grain growth [47-49,63] and liquid metal embrittlement [52, 53]. Generally, the formation of (relatively) disordered GB structures at high temperatures with enhanced transport properties can affect a broad range of GB-controlled materials properties, such as creep, corrosion and oxidation resistance, in addition to the microstructural evolution [14]. Furthermore, the GB structure and chemistry formed at the high processing temperature can often be (partially) retained upon cooling, thereby influencing a broad range of mechanical and physical properties at room temperature $[14,46]$.

The development of bulk phase diagrams and calculation of phase diagram (CALPHAD) methods established one of the foundations for modern materials science. Since GBs can exhibit transitions that can affect a broad range of fabrication processing and materials properties (as discussed above), it is useful to develop the GB analogues to bulk phase diagrams and CALPHAD methods. Recently, premelting and other types of GB transitions have been simulated by atomistic [57, 58], phase-fieldcrystal $[64,65]$ and lattice $[28,29]$ models; a limited number of GB complexion diagrams have been constructed by diffuse-interface models [20, 21] and a lattice model [37]. In a series of recent studies, bulk CALPHAD methods were extended to model GBs to forecast the stability of impurity-based, premelting-like IGFs in binary alloys; subsequently, a type of GB $\lambda$ diagrams have been developed to represent the thermodynamic tendency for general GBs to disorder and forecast related activated sintering behaviors in binary alloys (only) $[18,41,42,66]$. Although they are not yet rigorous GB complexion diagrams with well-defined transition lines and critical points, the correctness and usefulness of these binary GB $\lambda$ diagrams have been validated by a series of experiments. First, the model predictions were corroborated with direct HRTEM and GB chemistry analysis for selected systems [16, 18, 19, 42, 67]. 
Second, the computed GB $\lambda$ diagrams (with no free parameters) correctly predicted the onset sintering temperatures for a series of W based binary alloys as well as some trends in sintering rates $[18,42,66]$. Specifically, the predicted GB solidus temperature was consistent with a prior direct GB diffusivity measurement for $\mathrm{W}-\mathrm{Co}$ (the primary element/phase is underlined) using radioactive tracers [42, 68]. Third, the estimated GB diffusivity as a function of temperature and overall composition correlated well with the computed binary GB $\lambda$ diagram for Mo-Ni [18]. Finally, a counterintuitive phenomenon of decreasing GB diffusivity with increasing temperature was predicted and subsequently verified experimentally in a Mo +0.5 at. \% Ni alloy [54].

Historically, bulk CALPHAD methods were first developed for binary alloys, for which phase diagrams have already been determined by experiments. CALPHAD methods become useful when they can utilize the thermodynamic data that are largely obtained from binary systems to extrapolate (by adding only a few multi-body interaction parameters) and predict behaviors of multicomponent alloys, where the Edisonian approach is no longer feasible. Likewise, after demonstrating the basic feasibility of constructing binary GB $\lambda$ diagrams and their usefulness in predicting sintering behaviors, the challenging goal of this study is to extend and validate the model and computation methods to multicomponent $(N \geq 3)$ alloys. This work is not only technologically important (since understanding such interactions can offer a way to control GB behaviors via developing co-alloying strategies) but also scientifically interesting (because interactions among multiple alloying elements can produce new interfacial phenomena).

It should be noted that these computed $\lambda$ diagrams, which represent the thermodynamic tendency for average general GBs to disorder, are called "GB diagrams" in this article because they are not rigorous complexion diagrams with well-defined transition lines and critical points.

\section{The Model and Computational Methods}

\subsection{The Interfacial Thermodynamic Model}

In a phenomenological model adapted from the premelting and wetting models that were widely used by physicists $[3,64,69,70]$, the excess free energy of a subsolidus, premelting-like IGF is expressed as:

$$
\sigma^{x}(h)=\Delta G_{\text {amorph }}^{(\mathrm{vol})} h+2 \gamma_{\mathrm{cl}}+\sigma_{\text {interfacial }}(h),
$$

where $\Delta G_{\text {amorph }}^{(\mathrm{vol})}$ is the volumetric free energy for forming an undercooling liquid from the equilibrium solid phase(s), $h$ is the film thickness and $\gamma_{\mathrm{cl}}$ is the crystal-liquid interfacial energy. The interfacial 
potential, $\sigma_{\text {interfacial }}(h)$, includes the effects of all interfacial interactions and it vanishes as the film thickness $(h)$ approaches infinity (by definition, so that $2 \gamma_{\mathrm{cl}}$ can be well defined at $h \rightarrow+\infty$ ):

$$
\sigma_{\text {interfacial }}(+\infty)=0
$$

We may re-write the interfacial potential as:

$$
\sigma_{\text {interfacial }}(h)=-\Delta \gamma \cdot[1-f(h)]
$$

where $f(h)$ is defined as a dimensionless interfacial coefficient that satisfies:

$$
\left\{\begin{array}{l}
f(0)=0 \\
f(+\infty)=1
\end{array}\right.
$$

and

$$
\Delta \gamma \equiv 2 \gamma_{\mathrm{cl}}-\gamma_{G B}^{(0)}
$$

where $\gamma_{\mathrm{GB}}^{(0)} \equiv \sigma^{x}(0)$ represents the excess free energy of a hypothetic "dry" and "clean" GB without any adsorption and disorder (noting that $\gamma_{\mathrm{GB}}^{(0)}$ differs from the equilibrium $\gamma_{\mathrm{GB}}$ in general).

A subsolidus, premelting-like IGF can form spontaneously if the energy penalty for forming an undercooled quasi-liquid film can be overcompensated by the reduction of the interfacial energies:

$$
-\Delta \gamma \cdot f(h)>\Delta G_{a m o r p h}^{(v o l)} h
$$

Thus, we can introduce a thermodynamic parameter $[18,41,42,66]$ :

$$
\lambda \equiv \frac{-\Delta \gamma}{\Delta G_{\text {amorph }}^{(\text {vol) }}}
$$

which scales (but is not identical to) the actual (effective) interfacial width. Then, Eq. (1) can be rewritten in a dimensionless form, as follows:

$$
\frac{\sigma^{x}(h)-\sigma^{x}(0)}{\sigma_{\text {interfacial }}(+\infty)-\sigma_{\text {interfacial }}(0)}=\frac{\sigma^{x}(h)-\gamma_{G B}^{(0)}}{\Delta \gamma}=-\frac{h}{\lambda}+f(h) .
$$

At a thermodynamic equilibrium, an IGF will adopt an equilibrium thickness $\left(h_{\text {eq }}\right)$ that corresponds to the global minimum in Eq. (1) or Eq. (8). If the interfacial potential (coefficient) follows a simple exponentially decaying form, $h_{\mathrm{eq}}=\xi \ln (\lambda / \xi)$, where $\xi$ is a coherent length. In a general case, the interfacial 
potential, which includes multiple interfacial interactions that are often coupled, has a more complex form and is difficult to be precisely quantified. Nonetheless, the parameter $\lambda$ can be well defined and quantified to represent the thermodynamic tendency for average general GBs to disorder.

A key approximation for this approach is that a reference (average) film composition is used; in this work, the average film composition that maximizes $\lambda$ is adopted. In reality, thorough-thickness gradients in both composition and structure exist and are important character of GB complexions, which adopt the compositional and structural profiles that minimize the excess interfacial energies (so that they are thermodynamically 2-D interfacial phases); such gradients can be better modeled in diffuse-interface models $[20,21]$; yet, we note that the gradient energy terms can be treated approximately in the interfacial potential or coefficient $\left(\sigma_{\text {interfacial }}(h)\right.$ or $\left.f(h)\right)$ term in the current approach [14].

\subsection{Estimation of Reference Interfacial Energies}

The interfacial energies can be estimated by a macroscopic-atom model that was originally developed by Benedictus, Böttger, and Mittemeijer for simulating solid-state amorphization [71, 72], which utilizes the Miedema type parameters. Since we use CALPHAD data to compute bulk thermodynamic functions, here we adopt and refine a lattice model [73] that utilizes the regular-solution (pair-interaction) parameters to estimate interfacial energies to be self-consistent (as a further improvement from the prior studies $[18,41,42,66]$ that used the macroscopic-atom model, in addition to a major extension from binary to multicomponent alloys). In such a lattice model, the interfacial energy of an interface between a crystal of pure $A$ (with a negligibly small solubility of $B$ ) and a liquid of a uniform composition of $X_{B}^{L}$ (the molar/atomic fraction of $B$ ) in a binary $A-B$ system is estimated as:

$$
\gamma_{c l} \approx \gamma_{c l}^{(0)}=\frac{\Delta H_{A}^{\text {fuse }}}{\kappa m_{1}^{-1} V^{2 / 3}}+\frac{\Omega_{A-B}^{L}}{\kappa m_{1}^{-1} V^{2 / 3}}\left(X_{B}^{L}\right)^{2}+\frac{1.9 R T}{\kappa m_{1}^{-1} V^{2 / 3}}
$$

where $\Delta H_{A}^{\text {fuse }}$ is the fusion enthalpy of $A, \Omega_{A-B}^{L}$ is the molar liquid phase regular-solution parameter, $V$ is the molar volume (neglecting the thermal expansion for simplicity), $m_{1}$ is the fraction of bonds crossing the interface, and $\kappa$ is a geometric factor $\left(\kappa \equiv A_{m} / V^{2 / 3}\right.$, where $A_{m}$ is the area of one mole of atoms spread as a monolayer [73]). The value of $\mathrm{\kappa m}_{1}^{-1}$ depends the crystalline orientation of the interface: for example, $\kappa m_{1}^{-1} \approx 5 \times 10^{8}$ for $(100)$ planes for any cubic lattice and $\kappa m_{1}^{-1} \approx 3.7 \times 10^{8}$ for the closepacked (111) plane in an FCC lattice. In this study, we set $\kappa m_{1}^{-1}=C_{0} \approx 4.5 \times 10^{8}$ to represent the average 
value (of all different crystalline orientations), which is a parameter adopted in the macroscopic-atom model [74] to represent the average grain surface orientation of general GBs in a polycrystal.

The first term in Eq. (9) is an enthalpic contribution that corresponds to the excess enthalpy of a crystal-liquid interface of pure $A$. The second term represents the chemical interaction, a.k.a. the change in the interfacial energy when the fraction of $B$ in the liquid increases from zero to $X_{B}^{L}$, where bonds at the solid-liquid interface are assumed to be liquid type. The third term is an entropic contribution due to the near-interface ordering, which is adapted from the Benedictus-Böttger-Mittemeijer model [71, 72]. The superscript (0) is used in $\gamma_{c l}^{(0)}$ to denote that it is a reference interfacial energy without considering the near-interface variation in composition (as an estimation of the true equilibrium $\gamma_{c l}$ ). The main reason to adopt this lattice model is that it uses the regular-solution parameters that can be obtained from the CALPHAD data so that the same set of parameters are used in both the bulk thermodynamic functions and the statistic interfacial thermodynamic model to compute GB $\lambda$ diagrams in a self-consistent manner.

Eq. (9) can be further extended to model a multicomponent alloy, where the interfacial energy of an interface between a crystal of pure $A$ (with negligible solid solubilities) and a multicomponent liquid (of composition $\left.X_{i}^{L}, i=\mathrm{A}, \mathrm{B}, \mathrm{C} \ldots\right)$ is estimated as:

$$
\gamma_{c l} \approx \gamma_{c l}^{(0)}=\frac{1}{C_{0} V^{2 / 3}}\left[\Delta H_{1}^{\text {fuse }}+\left(\sum_{i \neq A} \Omega_{i-\mathrm{A}}^{L} X_{i}^{L}-\frac{1}{2} \sum_{i \neq j} \Omega_{i-j}^{L} X_{i}^{L} X_{j}^{L}\right)+1.9 R T\right]
$$

where the first, second, and third terms, respectively, are again the enthalpic, chemical, and entropic contributions, respectively, and $\kappa m_{1}^{-1}$ is set to be $C_{0}$. In Eq. (10), the $1 / 2 \sum_{i \neq j} \Omega_{i-j}^{L} X_{i}^{L} X_{j}^{L}$ term represents the reference energy state set by the chemical potential of the bulk liquid phase (noting that this term is not included for the Benedictus-Böttger-Mittemeijer model [71, 72], where the reference state is set to be the pure metals for solid state amorphization). A full derivation of Eq. (10) is given in Appendix A.

If the solid solubility limits are not negligibly small, Eq. (10) can be further generalized to:

$$
\gamma_{c l} \approx \gamma_{c l}^{(0)}=\frac{1}{C_{0} V^{2 / 3}}\left[\sum_{i} X_{i}^{C}\left(\Delta H_{i}^{f u s e}+\sum_{i \neq j} X_{j}^{L} \Omega_{i-j}^{L}\right)-\frac{1}{2}\left(\sum_{i \neq j} X_{i}^{L} X_{j}^{L} \Omega_{i-j}^{L}+\sum_{i \neq j} X_{i}^{C} X_{j}^{C} \Omega_{i-j}^{C}\right)+1.9 R T\right]
$$

where the superscripts "C" and " $\mathrm{C}$ " are used to represent the crystal and liquid phases, respectively. A full derivation of Eq. (11) is given in Appendix A. Eq. (11) can be reduced to Eq. (10) if $X_{A}^{C} \approx 1$ and $X_{i}^{C} \ll 1$ for all $i \neq \mathrm{A}$, which are the cases for most of our examples discussed below. 
To calculate the reference interfacial energy change $\Delta \gamma$ from Eq. (5), we also need to know the GB energy for a "dry" boundary (without any adsorption and disordering). If the solid solubility limits of all alloying elements are small, the measured GB energy of for the average general boundaries is typically used and the Turnbull estimation, $\gamma_{\mathrm{GB}}^{(0)} \approx 1 / 3 \gamma_{\mathrm{Surface}}^{(0)} \approx 1 / 3 \Delta H^{\text {vaporization }} /\left(C_{0} V^{2 / 3}\right)$, can be used in the cases where the experiment data are not available. If the solid solubility limits of alloying elements are not negligibly small, the "dry" GB energy of an alloy can be expressed as:

$$
\gamma_{G B}^{(0)} \approx \sum_{i} X_{i}^{C} \gamma_{G B, i}^{(0)}+\frac{Q}{C_{0} V^{2 / 3}} \sum_{i \neq j} X_{i}^{C} X_{j}^{C} \Omega_{i-j}^{C}
$$

where $Q$ is the average broken bond fraction, which is typically set to $1 / 6$ for an average general GB to satisfy the Turnbull estimation. Eq. (12) is also derived in Appendix A. In most case studies of W and Mo based alloys presented in this paper, the solid solubility limits are small so that Eq. (10) and experimentally measured GB energy of the average general boundaries can be used to estimate $\Delta \gamma$.

\subsection{Evaluating the Volumetric Free-Energy Penalty for Forming an Undercooled Liquid}

Bulk CALPHAD data (thermodynamic functions) and methods are used to evaluate the volumetric free energy penalty for forming an undercooled liquid, $\Delta G_{\text {amorph }}^{(\mathrm{vol})}$. The free energy of a phase $\Phi$ in a multicomponent system can be expressed as:

$$
G^{\Phi}=\sum_{i} X_{i}^{\Phi 0} G_{i}^{\Phi}+R T \sum_{i} X_{i}^{\Phi} \ln X_{i}^{\Phi}+{ }^{X S} G^{\Phi}
$$

where $X_{i}^{\Phi}$ and ${ }^{0} G_{i}^{\Phi}$, respectively, are the fraction and the free energy, respectively, of the component $i$. ${ }^{x S} G^{\Phi}$ is the excess free energy of mixing, which can be expressed in a Redlich-Kister polynomial:

$$
{ }^{X S} G^{\Phi}=\sum_{m} \sum_{i \neq j} L_{m(i, j)}^{\Phi} X_{i}^{\Phi} X_{j}^{\Phi}\left(X_{i}^{\Phi}-X_{j}^{\Phi}\right)^{m}
$$

Here, $L_{m(i, j)}^{\Phi}$ is the m-th order interaction parameter between the components $i$ and $j$ in the phase $\Phi$. Specifically, $L_{0(i, j)}^{\Phi}$ is the regular solution parameter $\omega_{l-j}^{\Phi}$ and this polynomial is reduced to a regularsolution equation for the zero-th order expansion ( $m=0$ only). The molar free-energy penalty for forming an undercooled liquid (film) of composition $\mathbf{X}_{f i l m}^{L}=\left\{X_{i}^{L}, i=A, B, C \ldots\right\}$ can be written as:

$$
\Delta G_{\mathrm{amorph}}^{(\mathrm{mol})}=G^{\mathrm{L}}\left(\mathbf{X}_{f i l m}^{L}\right)-\sum_{i} \mu_{i}^{C} X_{i}^{\mathrm{L}}
$$


The first term in Eq. (15) is the molar free energy of the bulk liquid phase. It is important to note that partial order and compositional gradients generally exist in the nanoscale quasi-liquid IGF so that it is not perfect liquid; however, $\lambda$ can be defined and calculated based on a reference state of uniform and perfect liquid film, while the variations in free energies due to order and compositional gradients can be (at least partially) considered in the interfacial potential term in Eq. (1); this approach was elaborated in a prior review [14]. The second term in Eq. (15) represents the reference free-energy state set by the chemical potentials $\left(\mu_{i}^{C}\right)$ of the bulk phase (crystalline grains) of a given composition $\mathbf{X}_{b u l k}^{C}=\left\{X_{i}^{C}, i=A, B, C \ldots\right\}$ :

$$
\sum_{i} \mu_{i}^{C} X_{i}^{\mathrm{L}}=G^{\mathrm{C}}\left(\mathbf{X}_{b u l k}^{C}\right)+\sum_{i \neq A} \frac{\partial G^{\mathrm{C}}}{\partial X_{i}}\left(X_{i}^{\mathrm{L}}-X_{0, i}^{C}\right)
$$

As illustrated in Fig. 1, $\Delta G_{\mathrm{amorph}}^{(\mathrm{mol})}$ is represented by the distance between the liquid free-energy surface and the bulk chemical potential plane at the composition of $\mathbf{X}_{\text {film }}^{L}$ in a ternary system. Then, the volumetric free-energy penalty for forming an undercooled liquid is given by:

$$
\Delta G_{\text {amorph }}^{(\mathrm{vol})}=\frac{\Delta G_{\mathrm{amorph}}^{(\mathrm{mol})}}{\sum_{i} X_{i}^{\mathrm{L}} V_{i}}
$$

Finally, Eq. (7) can be re-written for a multicomponent alloy as:

$$
\lambda\left(\mathbf{X}_{\text {bulk }}^{C}\right)=\operatorname{Max}_{\left\{\text {all possible } \mathbf{X}_{\text {film }}^{L}\right\}}\left\{\frac{-\left[2 \gamma_{c l}\left(\mathbf{X}_{\text {film }}^{L}, \mathbf{X}_{b u l k}^{C}\right)-\gamma_{G B}^{(0)}\left(\mathbf{X}_{b u l k}^{C}\right)\right]}{\Delta G_{\text {amorph }}^{(\mathrm{vol})}\left(\mathbf{X}_{\text {film }}^{L}, \mathbf{X}_{\text {bulk }}^{C}\right)}\right\} .
$$

Noting that in this work the average film composition $\left(\mathbf{X}_{\text {film }}^{L}\right)$ that maximizes $\lambda$ is adopted as a reference film composition. Subsequently, $\lambda$ can be computed as a function of the bulk composition $\left(\mathbf{X}_{b u l k}^{C}\right)$ and lines of constant $\lambda$ can be plotted in a bulk phase diagram to construct a GB $\lambda$ diagram.

\section{Construction of a Ternary GB $\lambda$ Diagram with All Phases}

\subsection{Ternary $\lambda$ Diagram for Average General GBs}

First, we use W-Ni-Fe (a.k.a. Ni and Fe co-doped/co-alloyed W, where the average, general GBs of the $\mathrm{W}$ primary phase are represented in the computed $\lambda$ diagram) as an example to demonstrate how to construct a ternary GB $\lambda$ diagram with multiple phases, including the secondary crystalline precipitates. Fig. 2(a) and (b) schematically illustrate a two-step procedure of constructing an isothermal section of a 
ternary GB $\lambda$ diagram. First, we computed $\lambda\left(\mathbf{X}_{\text {bulk }}^{B C C}\right)$ as a function of bulk composition $\left(\mathbf{X}_{b u l k}^{B C C}=\left\{X_{W}^{B C C}, X_{N i}^{B C C}, X_{F e}^{B C C}\right\}\right.$; noting that in this case the primary crystal phase is the W-rich bodycentered cubic or BCC phase so we substitute "C" with "BCC") using Eq. (18) and the bulk thermodynamic functions developed in Ref. [75, 76] at a constant temperature of $1673 \mathrm{~K}$. Subsequently, we plotted the computed $\lambda$ values (represented by the color) in the composition space of the W-Ni-Fe ternary system (for the W-rich corner) in Fig. 2(a), where we also plotted lines of constant $\lambda$ values. In this W-rich corner, $\lambda$ values increase monotonically with increasing $\mathrm{Ni}$ and Fe compositions, indicating that both $\mathrm{Ni}$ and Fe promote GB disorder.

In the second step, we considered the precipitation of secondary crystalline phases, such as the Nirich FCC phase and the $\mu$-FeW compound in this specific case, which limit the chemical potentials of $\mathrm{Ni}$ and Fe. The $\lambda$ values are determined by the chemical potentials of the bulk phase(s). Thus, in a ternary system, the $\lambda$ values are constant along the tie lines in any two-phase region, identical to the values at the solid solubility limit (solvus or solidus) line of the BCC alloy. Moreover, the $\lambda$ value is a constant throughout a three-phase coexistent region in a ternary alloy. Subsequently, the bulk phase boundaries and tie lines in two-phase regions ( $\mathrm{BCC}+\mathrm{FCC}$ and $\mathrm{BCC}+\mu$-FeW, respectively, in this specific case) were calculated for the $\underline{\mathrm{W}}-\mathrm{Ni}$-Fe system using bulk CALPHAD methods to construct the equilibrium ternary GB $\lambda$ diagram with all equilibrium bulk phases, which is shown in Fig. 2(b).

\subsection{GB-to-GB Variations}

We emphasize that computed GB $\lambda$ diagrams represent the average behaviors of general GBs and recognize significant GB-to-GB variations in polycrystalline alloys because GBs have five macroscopic degrees of freedom. Such GB-to-GB variations may be modeled by assuming the initial $\gamma_{\mathrm{GB}}^{(0)}$ can vary by approximately $\pm 15 \%$ due to the anisotropy (which is typically for the general GBs for most metals). Fig. 3(b) shows a computed GB $\lambda$ diagram for $\underline{\mathrm{W}}-\mathrm{Ni}$-Fe that considers GB-to-GB variations, where the lines of constant $\lambda$ (in Fig. 3(a)) expand to bands (in Fig. 3(b)); noting that these bands are only to represent the variations in general GBs, while the special low-energy GBs are not represented here. Such GB-to-GB variations may be a root cause for abnormal grain growth, as hypothesized in a series of prior studies by Harmer and co-workers $[48,49,63]$, and our calculations support and quantitatively rationalize this hypothesis. Although the lines of constant $\lambda$ should always be bands for any polycrystal, we typically only plot GB $\lambda$ diagrams that reflect the average general $G B s$ to ensure the clarity of the diagram. It is important to recognize that GB-to-GB variations similar to that shown in Fig. 3(b) ubiquitously exist in all $\mathrm{W}$ and Mo based GB $\lambda$ diagrams computed in this paper. 


\section{Effects of Adding Co-Alloying Elements on GB disorder in Ternary Systems}

Following earlier experimental and modeling studies of the binary $\underline{\mathrm{W}}-\mathrm{Ni}[15,16,42,66]$ and $\underline{\mathrm{Mo}}-\mathrm{Ni}$ $[18,19,54]$ alloys, we first conducted numerical experiments of $\underline{\mathrm{W}}-\mathrm{Ni}-X$ and $\underline{\mathrm{Mo}}-\mathrm{Ni}-X$ alloys (with $X$ being a fictive element) to identify the key thermodynamic parameters that influence co-alloying effects. Specifically, we systematically varied five key parameters, i.e., the melting temperature of $X\left(T_{X}^{m}\right)$ and four regular-solution parameters $\left(\Omega_{X-A}^{\mathrm{L}}, \Omega_{X-A}^{\mathrm{BCC}}, \Omega_{X-\mathrm{Ni}}^{\mathrm{L}}, \Omega_{X-\mathrm{Ni}}^{\mathrm{BCC}}\right.$, where $A=\mathrm{W}$ or Mo, assuming that $X$ forms regular solutions with $\mathrm{W}, \mathrm{Mo}$ and $\mathrm{Ni}$ in both solid and liquid phases). To focus on investigating the effects of these key parameters, a few simplifications were adopted. We assumed the fusion entropy of $X$ ( $\Delta S_{X}^{\text {fuse }}$ ) to be10 $\mathrm{J} / \mathrm{mol} \cdot \mathrm{K}$ and its molar volume $\left(V_{\mathrm{X}}\right)$ to be $8 \mathrm{~cm}^{3} / \mathrm{mol}$ (both are typical values for transition metals). Moreover, we only considered the BCC and liquid phases in these calculations (while the precipitation of other phase(s) was considered in examples of real alloys in the next two sections).

Prior studies had already demonstrated the formation of $0.80 \pm 0.12 \mathrm{~nm}$ thick Ni-enriched quasi-liquid IGFs in Mo $+1 \% \mathrm{Ni}$ specimens at $1668 \mathrm{~K}$ ( $v s$. the computed $\lambda=0.99 \mathrm{~nm})[18,19]$. In

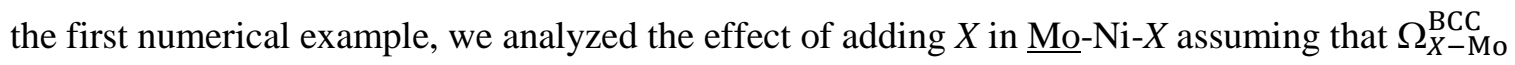
$= \pm 25 \mathrm{~kJ} / \mathrm{mol}, \Omega_{X-\mathrm{Mo}}^{\mathrm{L}}=\Omega_{X-\mathrm{Ni}}^{\mathrm{L}}=\Omega_{X-\mathrm{Ni}}^{\mathrm{BCC}}=0$ and $T_{X}^{m}=2000 \mathrm{~K}$ and using the Mo-Ni binary CALPHAD data from Ref. [77]. The computed GB $\lambda$ diagrams shown in Fig. 4 suggest that a positive regular-solution (pair-interaction) parameter $\left(\Omega_{X-\text { Mo }}^{\mathrm{BCC}}\right)$ with Mo in the solid phase promotes GB disorder (by rejecting $X$ into the IGFs, as evident in the film compositional map in Fig. 4). In comparison, a negative $\Omega_{X-\text { Mo }}^{\mathrm{BCC}}$ slightly inhibits GB disorder; the corresponding film compositional map shows that $X$ does not segregate to GBs/IGFs appreciably. It is interesting to note that in the W-Ni-Fe example shown in Fig. 2, co-alloying of Fe also enhances GB disordering in $\underline{\mathrm{W}}-\mathrm{Ni}$ systems as a result of a positive $\Omega_{\mathrm{Fe}-\mathrm{W}}^{\mathrm{BCC}}(=41.5 \mathrm{~kJ} / \mathrm{mol}$, while all other $\Omega$ 's are small; see Table I for all relevant parameters; the same effect was also evident in Fig. 8 for a lower temperature, where the co-alloying effect was confirmed by sintering experiments).We believe that this represents one general co-alloying effect in ternary systems.

Furthermore, we conducted a series of systematic numerical experiments of $\underline{\mathrm{W}}-\mathrm{Ni}-X$ systems, where the stabilization of subeutectic quasi-liquid IGFs in the W-Ni system had been confirmed experimentally (Fig. 2(e)) $[15,16]$. In these calculations, we adopted $\gamma_{\mathrm{GB}}^{(0)}=1.08 \mathrm{~J} / \mathrm{m}^{2}$ using the experimental data reported in Ref. [78] and used the W-Ni binary thermodynamic functions developed in Ref. [76]. Then, we examined the effects of five key parameters $\left(T_{X}^{m}, \Omega_{X-\mathrm{W}}^{\mathrm{BCC}}, \Omega_{X-\mathrm{W}}^{\mathrm{L}}, \Omega_{X-\mathrm{Ni}}^{\mathrm{BCC}}\right.$ and $\Omega_{X-\mathrm{Ni}}^{\mathrm{L}}$; typical $\Omega$ values for $\mathrm{W}$ based systems are shown in Table I) via a systematical approach. 
First, we investigated the effect of the melting temperature of the co-alloying element $X$, where we set the four regular-solution parameters $\left(\Omega_{X-\mathrm{W}}^{\mathrm{BCC}}, \Omega_{X-\mathrm{W}}^{\mathrm{L}}, \Omega_{X-\mathrm{Ni}}^{\mathrm{BCC}}\right.$ and $\left.\Omega_{X-\mathrm{Ni}}^{\mathrm{L}}\right)$ to be zero for simplicity. Intuitively, one might speculate that adding a co-alloying element $X$ with a low melting temperature would promote the disordering of W GBs. However, the computed $\lambda$ diagrams shown in Fig. 5 suggest that reducing the melting temperature of $X$ has little effects on promoting GB disordering in the W-rich BCC phase.

Second, we varied one of the four regular-solution parameters $\left(\Omega_{X-\mathrm{W}}^{\mathrm{BCC}}, \Omega_{X-\mathrm{W}}^{\mathrm{L}}, \Omega_{X-\mathrm{Ni}}^{\mathrm{BCC}}\right.$ and $\Omega_{X-\mathrm{Ni}}^{\mathrm{L}}$, respectively), where we set $T_{m}^{X}$ to be $2000 \mathrm{~K}$ and the three other $\Omega$ 's to be zero. Fig. 6(a) and 6(b) show that a positive $\Omega_{X-\mathrm{W}}^{\mathrm{BCC}}$ or negative $\Omega_{X-\mathrm{W}}^{\mathrm{L}}$ promotes the GB disordering in $\mathrm{W}$ and a negative $\Omega_{X-\mathrm{W}}^{\mathrm{BCC}}$ or positive $\Omega_{X-\mathrm{W}}^{\mathrm{L}}$ suppresses GB disordering. This can be understood intuitively since a positive $\Omega_{X-\mathrm{W}}^{\mathrm{BCC}}$ should reject $X$ from the crystalline BCC phase, while a negative $\Omega_{X-\mathrm{W}}^{\mathrm{L}}$ should attract $X$ to GBs to form a liquid-like complexion (or a bulk secondary liquid phase above the solidus line when a sufficiently high fraction of $X$ is added). Since it is known that Ni segregates at GBs, which leads to the formation of liquid-like IGFs at high temperatures (but below the bulk solidus line), a negative $\Omega_{X-\mathrm{Ni}}^{\mathrm{L}}$ should promote GB disordering via attracting $X$ to the liquid-like GB structures to thicken the effective interfacial width (Fig. 6(d1)). Finally, while adding $X$ with a negative $\Omega_{X-N i}^{B C C}$ does suppress GB disordering (Fig. 6(c1)), a positive $\Omega_{X-\mathrm{Ni}}^{\mathrm{BCC}}$ does not appreciably enhance GB disordering as the case of positive $\Omega_{X-\mathrm{W}}^{\mathrm{BCC}}$ (as shown in Fig. 6(c4) vs. Fig. 6(a4)). This is presumably because the Ni content in the bulk phase is low, so that a positive $\Omega_{X-\mathrm{Ni}}^{\mathrm{BCC}}$ cannot effectively reject $X$ into GBs in the case of a positive $\Omega_{X-\mathrm{W}}^{\mathrm{BCC}}$ ). In summary, this set of numerical experiments (Fig. 6) suggest interesting GB adsorption and disordering behaviors that can be understood via the interactions of two co-alloying elements ( $\mathrm{Ni}$ and $X$ ) the primary metal $(\mathrm{W})$ and we should be able to generalize these understandings to other ternary alloys.

In general, the regular-solution parameters of the solid and liquid phases are not independent to each other; they are positively correlated and the regular-solution parameter of the corresponding solid is typically greater due to the additional strain energy in the solid phase (see typical values in Table I). Thus, in the last set of numerical experiments, we assumed $\left(\Omega_{\mathrm{W}-X}^{\mathrm{BCC}}-\Omega_{\mathrm{W}-X}^{\mathrm{L}}\right)=50 \mathrm{~kJ} / \mathrm{mol}$ (a mediate value) and varied $\Omega_{\mathrm{W}-X}^{\mathrm{L}}\left(\Omega_{\mathrm{W}-X}^{\mathrm{BCC}}\right)$. As shown in Fig. 7, addition of $X$ with a greater (coupled solid/liquid) regularsolution parameter promotes GB disordering, which represents another general co-alloying effect.

\section{Application to $\underline{\mathrm{W}}-\mathrm{Ni}-\mathrm{M}(\boldsymbol{M}=\mathrm{Fe}, \mathrm{Co}, \mathrm{Cr}, \mathrm{Zr}, \mathrm{Nb}$ and $\mathrm{Ti})$ Ternary Systems}


We further modeled W-Ni- $M(M=\mathrm{Fe}, \mathrm{Co}, \mathrm{Cr}, \mathrm{Zr}, \mathrm{Nb}, \mathrm{Ti})$ ternary systems. These real ternary alloys are generally more complex because the bulk crystal/liquid solutions are not just symmetrical regular solutions (with non-zero order terms in the Redlich-Kister polynomials) and various intermediate compounds can precipitate and change the bulk phase equilibria. We considered these effects via conducting full CALPHAD analyses of the bulk phases.

We followed earlier studies $[18,41,42,54]$ to use activated sintering experiments as an efficient way to test computed results (with the underlying assumption that the enhanced sintering rates are correlated with the GB structural disorder represented by computed $\lambda$ values, which has been proven in prior studies $[18,41,42,54])$. Specifically, specimens of $\mathrm{W}, \mathrm{W}-0.5 \mathrm{Ni}, \mathrm{W}-1 \mathrm{Ni}$ and $\mathrm{W}-0.5 \mathrm{Ni}-0.5 \mathrm{M}$ (at. \%) were made by mixing high-purity tungsten $(99.999 \%, \sim 5 \mu \mathrm{m}$ particle size) powder and metal chlorides (purchased from Alfa Aesar) in acetone solutions. Slurries were dried in an oven at $353 \mathrm{~K}\left(80^{\circ} \mathrm{C}\right)$ and calcined and reduced at $873 \mathrm{~K}$ for an hour in a tube furnace a flowing $\mathrm{Ar}+5 \% \mathrm{H}_{2}$ gas. The dried powders were then pressed into discs and pre-sintered at $1173 \mathrm{~K}$ for 2 hours in a flowing $\mathrm{Ar}+5 \% \mathrm{H}_{2}$ gas. After measuring the initial density $\left(\rho_{0}\right)$, the pre-sintered specimens were sintered isothermally at $1573 \mathrm{~K}$ for two hours in a flowing $\mathrm{Ar}+5 \% \mathrm{H}_{2}$ gas. The sintering experiments were conducted using a specially-designed vertical furnace where the specimens could be inserted into or withdrawn from the hot zone within $\sim 1$ minute so that the densification during ramping and cooling stages can be neglected [18]. The density of the sintered sample $(\rho)$ was measured to calculate the density increase percentage, $\left(\rho-\rho_{0}\right) / \rho$. The mean values measured from multiple specimens sintered at identical conditions were reported and the standard deviations were used as the error bars.

Fig. 8(a) shows the average density increase percentages for three compositions, pure $\mathrm{W}, \mathrm{W}-0.5 \mathrm{Ni}$ and $\mathrm{W}-0.5 \mathrm{Ni}-0.5 \mathrm{Fe}$ (at. \%), that were sintered at $1573 \mathrm{~K}$ for two hours. Addition of 0.5 at. $\%$ of Ni substantially increased the densification of $\mathrm{W}$, which was attributed to the enhanced mass transport in the subeutectic quasiliquid IGFs by both modeling and experimental studies [15, 16, 42, 66], and such Nibased disordered IGFs have been directly observed by HRTEM (Fig. 2(e)) [15, 16]. As shown in Fig. 8(a), adding 0.5 at. $\%$ of Fe together with 0.5 at. \% of Ni further enhanced sintering. To explain this coalloying effect, a ternary W-Ni-Fe GB $\lambda$ diagram was computed and shown in Fig. 8(b), where the three relevant composition points are labeled. Fig. 8(b) suggests that co-allying of Fe can promote GB disordering, which is consistent with experimental observations (Fig. 8(a)). The relevant regular-solution parameters of $\underline{\mathrm{W}}-\mathrm{Ni}-\mathrm{Fe}$ are shown in Table $\mathrm{I}\left(\Omega_{X-\mathrm{W}}^{\mathrm{BCC}}=41.5 \mathrm{~kJ} / \mathrm{mol}\right.$ and the other three are all small $)$; this case is close to the case shown in Fig. 6(a4). Thus, the enhancement effect is likely due to the large, positive $\Omega_{X-\mathrm{W}}^{\mathrm{BCC}}$, but the current case is more complex (than that shown in Fig. 8(a)) due to the formation of secondary FCC phase (which was represented in Fig. 6) and the W-Fe binary solutions are more 
complex than simple regular solutions (with high-order, asymmetrical terms in the Redlich-Kister polynomials).

Furthermore, we systematically computed ternary GB $\lambda$ diagrams for $\mathrm{W}-\mathrm{Ni}-M(M=\mathrm{Cr}, \mathrm{Zr}, \mathrm{Co}, \mathrm{Fe}$, $\mathrm{Nb}$ and $\mathrm{Ti}$ ) systems at $1573 \mathrm{~K}$ using the CALPHAD data from Refs. [75, 76, 79-86], where ternary compounds were represented whenever the relevant data exist; otherwise, the ternary systems were built by combining two binary systems. First, the computed ternary GB $\lambda$ diagrams with only the BCC and liquid phases (without considering the precipitation of any secondary crystalline phase) are shown in Fig. 9(a). Second, the computed ternary GB $\lambda$ diagrams considering all possible phases (or all phases with known thermodynamic data) are shown in Fig. 9(b). Finally, Fig. 9(c) shows the measured density increases after 2-hour isothermal sintering of various ternary $\mathrm{W}-0.5 \mathrm{Ni}-0.5 \mathrm{M}$ (at. \%) alloys, along with binary $\mathrm{W}-0.5 \mathrm{Ni}$ and $\mathrm{W}-1 \mathrm{Ni}$ (at. \%) alloys as the references; the measured densifications for these systems generally correlate with calculated $\lambda$ values with the exception of $\mathrm{W}-0.5 \mathrm{Ni}-0.5 \mathrm{Zr}$ (which will be explained separately). In fact, adding $\mathrm{Nb}$ and Ti likely enhanced the densification via liquid phase sintering since the compositions appeared to be above the solidus lines. Fe is the most effective solid-state co sintering aid, where the enhancement was likely due to the large, positive $\Omega_{X-\mathrm{W}}^{\mathrm{BCC}}$ (as we have discussed earlier). The computed results predicted $\mathrm{Co}$ and $\mathrm{Cr}$ to be less effective co sintering aids, consistent with experimental observations.

It is interesting to note that adding $\mathrm{Zr}$ decreased the sintering rate in $\mathrm{W}-0.5 \mathrm{Ni}-0.5 \mathrm{Zr}$, as compared with $\mathrm{W}-0.5 \mathrm{Ni}$ and $\mathrm{W}-1 \mathrm{Ni}$, although the computed $\lambda$ values suggest a small enhancement effect after adding $\mathrm{Zr}$ (at the thermodynamic equilibrium with the FCC and $\mathrm{ZrW}_{2}$ precipitation; Fig. 9(b2)). However, the computed GB $\lambda$ diagram for $\underline{\mathrm{W}}-\mathrm{Ni}-\mathrm{Zr}$ without the $\mathrm{FCC}$ and $\mathrm{ZrW}_{2}$ precipitation (Fig. 9(a2)) did suggest that adding $\mathrm{Zr}$ could suppress GB disordering if the precipitation was hindered, which offers a possible explanation for this exception. We note that $\mathrm{Zr}$ is prone to oxidation, which may be an alternative reason for $\mathrm{Zr}$ to suppress activated sintering.

\section{Applications to Mo-Si-B-M ( $M=\mathrm{Fe}, \mathrm{Co}$ and Ni) Quaternary Systems}

As the final application example, we used our model and computed GB $\lambda$ diagrams to select sintering aids to enhance the densification of Mo-Si-B based alloys. A prior experimental study reported the effects of various sintering aids on enhancing the densification of Mo-8.9Si-7.7Bi (at. \%) three-phase alloys, which contain the Mo-rich BCC, $\mathrm{A} 15\left(\mathrm{Mo}_{3} \mathrm{Si}\right)$ and $\mathrm{T} 2\left(\mathrm{Mo}_{5} \mathrm{SiB}_{2}\right)$ phases [17]. Cochran and co-workers originally introduced a reactive sintering method to sinter Mo-Si-B alloys [87]. Jung et al. found that adding 0.5 at. \% of Ni, Co and Fe can further enhance the densification (Fig. 10(a)), and the effectiveness was ranked as: $\mathrm{Ni}>\mathrm{Co}>\mathrm{Fe}[17]$. At that time, the methods to compute ternary and quaternary GB $\lambda$ 
diagrams had not been developed, so that the experimental results were explained based on binary interactions on a qualitative basis in Ref. [17]. Here, we computed the relevant ternary and quaternary GB $\lambda$ diagrams to further explain the effects of these sintering aids on a more quantitative basis.

First, we computed a ternary GB $\lambda$ diagram for Mo-Si-B at $1873 \mathrm{~K}$ (the sintering temperature), where we used CALPHAD data of the Mo-Si-B ternary system reported in Ref. [88-90] and estimated average general GB energy $\left(\gamma_{\mathrm{GB}}^{(0)}\right)$ for pure Mo to be $1 \mathrm{~J} / \mathrm{m}^{2}$ using the Turnbull estimation [78]. As shown in Fig. 10(b), the GBs in Mo-Si-B are highly "dry;” the computed $\lambda$ value generally increases with increase fraction of $\mathrm{Si}$ and reaches a maximum at $\lambda \approx 0.55 \mathrm{~nm}$ in the Mo-A15- $\mathrm{T}_{2}$ three-phase region. It should be noted that the computed $\lambda$ value in this three-phase region represents the average Mo general GBs in Mo8.9Si-7.7 $\mathrm{Bi}$, because the chemical potentials in the three-phase region are constant regardless the phase fractions . This result is consistent with the general understanding that Mo-Si-B alloy is difficult to sinter at this temperature [91] (and the reactive sintering route [87] has to be used to achieve $>90 \%$ of the theoretical density (Figs. 10(a) and (f))).

Subsequently, we computed pseudo-ternary sections of the GB $\lambda$ diagrams for the Mo-Si-B-0.5M ( $M$ $=\mathrm{Ni}, \mathrm{Co}$ and $\mathrm{Fe}$ ) systems at $1873 \mathrm{~K}$ and a fixed atomic fraction of $0.5 \% M$, by combining the Mo-Si-B ternary CALPHAD data [88] with Mo- $M, M$-Si, $M$-B binary thermodynamic functions adopted from Refs.[77, 92-98]. Here, we adopted two simplifications, as follows: (1) $M$ has negligible solubility in $\mathrm{T}_{2}$ or A15 phase and (2) no $M$-containing ternary or quaternary compounds precipitates in the composition region (up to 0.5 at. \% $M$ ). The computed pseudo-ternary sections of the GB $\lambda$ diagrams for $\underline{\mathrm{Mo}}-\mathrm{Si}$-B$0.5 \mathrm{Ni}, \underline{\mathrm{Mo}}-\mathrm{Si}-\mathrm{B}-0.5 \mathrm{Co}$, and $\underline{\mathrm{Mo}}-\mathrm{Si}-\mathrm{B}-0.5 \mathrm{Fe}$ GB are shown in Fig. 10(c-e). It can be found that the computed $\lambda$ values increased significantly (reaching $\lambda>2 \mathrm{~nm}$ in the Mo-A15-T $\mathrm{T}_{2}$ three-phase region, which corresponds to the chemical potentials of Mo GBs during the sintering) after adding 0.5 at. \% Ni.

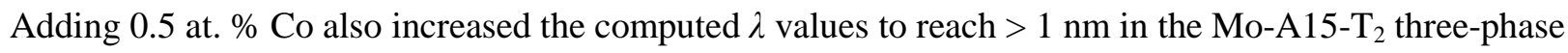
region, while adding 0.5 at. $\% \mathrm{Fe}$ increased the computed $\lambda$ values moderately. Thus, the computed $\lambda$ diagrams can correctly predict the relative effectiveness of these three sintering aids $(\mathrm{Ni}>\mathrm{Co}>\mathrm{Fe})$. With the best sintering aid Ni, the densification could be substantially improved after sintering at $1873 \mathrm{~K}$ for 12 hour to reach $\sim 97 \%$ of the theoretical density (Fig. 10 (g)), which represents the highest sintered density achieved for this alloy. It should be noted that nanometer-thick, impurity-based, disordered (quasi-liquid) IGFs have been directly observed by HRTEM in sintered Mo-Si-Ni alloys in the prior study [17], suggesting that enhanced densification is due to increased transport in these quasi-liquid IGFs.

\section{Conclusions}


A quantitative interfacial thermodynamic model has been developed for computing GB $\lambda$ diagrams for ternary and quaternary alloys to forecast useful trends in GB disordering and related sintering (and potentially a wide range of other GB-controlled) properties. Numerical experiments have been conducted for Mo-Ni- $X$ and $\underline{\mathrm{W}}-\mathrm{Ni}-X$ systems to identify the key thermodynamic parameters that control the GB disordering behaviors. Subsequently, the model and computation methods have been applied to $\underline{\mathrm{W}}-\mathrm{Ni}-M$ $(M=\mathrm{Fe}, \mathrm{Nb}, \mathrm{Ti}, \mathrm{Cr}, \mathrm{Zr}$ and $\mathrm{Co})$ and $\underline{\mathrm{Mo}}-\mathrm{Si}-\mathrm{B}-M(M=\mathrm{Ni}, \mathrm{Co}$ and $\mathrm{Fe})$ systems. It has been demonstrated that the computed GB $\lambda$ diagrams can predict some useful trends in the relative effectiveness of various sintering aids.

In addition to the derivation and validation of the model for $N \geq 3$ systems, a further contribution of the current work is the derivation of a set of equations to use CALPHAD-derived data to estimate interfacial energies, which represent a more consistent and accurate approach than those used in prior studies of binary alloys $[18,41,42,66]$, where the same set of CALPHAD based parameters can be used in both bulk and interfacial thermodynamic computations is a self-consistent manner.

In general, it is important to develop GB diagrams for multicomponent alloys, where the Edisonian approach is no longer valid to select the optimal combination of multiple alloying elements. Practically, multicomponent GB $\lambda$ diagrams can be used to understand the interactions of multiple alloying elements at GBs, thereby developing co-alloying strategies to control GBs. It should be emphasize that GB $\lambda$ diagrams are not yet rigorous GB "phase" (complexion) diagrams with well-defined transition lines/curves. Nonetheless, multicomponent GB $\lambda$ diagrams can be used to forecast some useful trends in GB segregation and disordering, representing an important step towards a long-range scientific goal of developing interfacial "phase" (complexion) diagrams as a general materials science tool, which can be used to help accelerating materials design, as well as achieving predictive fabrication by design, in the spirit of the Materials Genome Initiative [41, 46, 66].

Acknowledgements: This work was primarily supported by the Aerospace Materials for Extreme Environments program of the U.S. Air Force Office of Scientific Research (under the AFOSR grants No. FA9550-10-1-0185 for 05/2010 to 04/2013 and No. FA9550-14-1-0174 after 09/2014), and we gratefully thank our AFOSR program manager, Dr. Ali Sayir, for his consistent support and guidance. We also acknowledge partial supports from DOE-NETL (DE-FE0011291 for studying the creep of W alloys) and NSF (CMMI-1436305 for studying the sintering of W alloys) for extending this work to W based alloys, particularly during the AFOSR funding gap. J.L. acknowledges the partial support from an National Security Science and Engineering Faculty Fellowship (ONR grant no. N00014-15-1-0030) to further extend the work of developing interfacial "phase" diagrams to other metallic and ceramic systems to 
reach the proposed long-range scientific goal. We thank Zhao Zhang for providing unpublished experimental data on sintering of W-Ni-M systems (Fig. 9(c)) and Dr. Jae-Il Jung for providing experimental data on sintering of Mo-Si-B-M (including Fig. 10(a) that was re-plotted from Ref. [17] as well as the sintering data and SEM images shown in Fig. 10 ( $\mathrm{f}$ and $\mathrm{g}$ ) that have not been published before) to be compared with the modeling results. 


\section{Appendix A:}

\section{Derivations of Interfacial Energies in a Statistical Multicomponent Lattice Model}

The interfacial bonds at a crystal-liquid interface are assumed to be liquid type. This is the assumption used in both the Benedictus-Böttger-Mittemeijer model [71, 72] and the prior binary lattice model [73]. The total energy for all cross-interface bonds is:

$$
\sigma_{c l}=z_{v} N_{0}\left(\sum_{i} X_{i}^{L} X_{i}^{C} e_{i-i}^{L}+\sum_{i \neq j} X_{i}^{L} X_{j}^{C} e_{i-j}^{L}\right)
$$

where $X_{\mathrm{i}}$ is the atomic fraction of the component $i(i=\mathrm{A}, \mathrm{B}, \mathrm{C} \ldots), e_{\mathrm{i}-\mathrm{j}}$ represents the bond energy between the atoms $i$ and $j, z_{\mathrm{v}}$ is the coordination number (number of bonds per atom) across the interface, $N_{0}$ is the number of atoms per unit area at the interface, and the superscripts denote the crystal (C) or liquid (L) phase. Since the pair-interaction parameter (i.e. the regular-solution parameter per bond) is defined as $\omega_{i-j}^{L}=e_{i-j}^{L}-1 / 2\left(e_{i-i}^{L}+e_{j-j}^{L}\right)$, Eq. (A1) can be rewritten as:

$$
\sigma_{c l}=z_{v} N_{0}\left[\frac{1}{2}\left(\sum_{i} X_{i}^{S} e_{i-i}^{L}+\sum_{i} X_{i}^{L} e_{i-i}^{L}\right)+\sum_{i \neq j} X_{i}^{L} X_{j}^{S} \omega_{i-j}^{L}\right]
$$

The interfacial energy $\gamma_{c l}^{(0)}$ (where the superscript "(0)" denotes no interfacial adsorption) is the excess bond energies for the crystal-liquid interface plus an entropic contribution originated from ordering near the interface:

$$
\gamma_{c l}^{(0)}=\left[\sigma_{c l}-\frac{1}{2}\left(\sigma_{l l}+\sigma_{c c}\right)\right]+\frac{1.9 R T}{C_{0} V^{2 / 3}}
$$

where $\sigma_{\mathrm{cc}}$ and $\sigma_{\mathrm{ll}}$, respectively, are the total energy for the cross-interface bonds in a plane of identical geometry in the bulk crystal and liquid phases, respectively, which can be expressed as:

$$
\left\{\begin{array}{l}
\sigma_{l l}=z_{v} N_{0}\left(\sum_{i} X_{i}^{L} e_{i-i}^{L}+\sum_{i \neq j} X_{i}^{L} X_{j}^{L} \omega_{i-j}^{L}\right) \\
\sigma_{c c}=z_{v} N_{0}\left(\sum_{i} X_{i}^{C} e_{i-i}^{C}+\sum_{i \neq j} X_{i}^{C} X_{j}^{C} \omega_{i-j}^{C}\right)
\end{array} .\right.
$$

Eq. (A4) can be derived via similar routes as that used to deduce Eq. (A2). Moreover, we have the following basic relations: 


$$
\left\{\begin{array}{l}
m_{1}=\frac{z_{v}}{z} \\
\frac{N_{0}}{N_{\text {Avogadro }}}=\frac{1}{\kappa V^{2 / 3}} \\
\kappa m_{1}^{-1}=C_{0} \quad(\text { for an average general GB }), \\
\Delta H_{i}^{\text {fuse }}=\frac{z}{2} N_{\text {Avogadro }}\left(e_{i-i}^{L}-e_{i-i}^{S}\right) \\
\Omega_{i-j}=z N_{\text {Avogadro }} \omega_{i-j}
\end{array}\right.
$$

where $N_{\text {Avogadro }}$ is the Avogadro number, $z$ is the total coordination number, $\Omega_{\mathrm{i}-\mathrm{j}}$ is molar regular-solution parameter, and $m_{1}, \kappa$, and $C_{0}$ are parameters defined in the main text. Combining Eqs. (A2)- (A5) produces:

$$
\gamma_{c l}^{(0)}=\frac{1}{C_{0} V^{2 / 3}}\left[\sum_{i} X_{i}^{C}\left(\Delta H_{i}^{\text {fuse }}+\sum_{i \neq j} X_{j}^{L} \Omega_{i-j}^{L}\right)-\frac{1}{2}\left(\sum_{i \neq j} X_{i}^{L} X_{j}^{L} \Omega_{i-j}^{L}+\sum_{i \neq j} X_{i}^{C} X_{j}^{C} \Omega_{i-j}^{C}\right)+1.9 R T\right],
$$

which is Eq. (11) in the main text. Eq. (10) in the main text can be obtained by assuming $X_{A}^{C}=1$ and $X_{i}^{C}=0$ for all $i \neq A$.

Similarly, the "dry" GB energy (without any adsorption and disorder) for a multicomponent alloy can be estimated as:

$$
\gamma_{G B}^{(0)} \approx Q z_{v} N_{0}\left[\sum_{i} X_{i}^{C} e_{i-i}+\sum_{i \neq j} X_{i}^{C} X_{j}^{C} \omega_{i-j}\right]
$$

where $Q$ is the average broken bond fraction at the general GBs, which is typically set to $1 / 6$ for an average general GB so that the Turnbull estimation, $\gamma_{\mathrm{GB}}^{(0)} \approx 1 / 3 \gamma_{\mathrm{Surface}}^{(0)}$, holds. Since the average GB energy for general GBs in pure $i$ can be estimated as $\gamma_{G B, i}^{(0)} \approx Q z_{v} N_{0} e_{i i}$, Eq. (A7) can be rewritten as:

$$
\gamma_{G B}^{(0)} \approx \sum_{i} X_{i}^{C} \gamma_{G B, i}^{(0)}+\frac{Q}{C_{0} V^{2 / 3}} \sum_{i \neq j} X_{i}^{C} X_{j}^{C} \Omega_{i j}^{C}
$$

which is Eq. (12) in the main text. We should note that experimentally-measured GB energies $\left(\gamma_{G B, i}^{(0)}\right)$ should be used (instead of the Turnbull estimations), if the data are available. 
Table I. The regular solution parameters for $\mathrm{W}-M$ and $\mathrm{Ni}-M(M=\mathrm{Ni}, \mathrm{Co}, \mathrm{Fe}, \mathrm{Zr}, \mathrm{Cr}$, Ti and $\mathrm{Nb})$ at $1573 \mathrm{~K}$ from Ref. $[75,76,79-86]$. The datum for $\Omega_{\mathrm{Ni}-\mathrm{Co}}^{\mathrm{BCC}}$ is not available so that $\Omega_{\mathrm{Ni}-\mathrm{Co}}^{\mathrm{FCC}}$ is listed instead (denoted by ${ }^{*}$ ).

\begin{tabular}{|c|c|c|c|c|}
\hline$M=$ & $\Omega_{\mathrm{W}-M}^{\mathrm{BCC}}(\mathrm{kJ} / \mathrm{mol})$ & $\Omega_{\mathrm{W}-M}^{\mathrm{L}}(\mathrm{kJ} / \mathrm{mol})$ & $\Omega_{\mathrm{Ni}-M}^{\mathrm{BCC}}(\mathrm{kJ} / \mathrm{mol})$ & $\Omega_{\mathrm{Ni}-M}^{\mathrm{L}}(\mathrm{kJ} / \mathrm{mol})$ \\
\hline $\mathrm{Ni}$ & 82.0 & -0.9 & $/$ & $/$ \\
\hline $\mathrm{Co}$ & 54.7 & -16.7 & $1.3^{*}$ & 1.3 \\
\hline $\mathrm{Fe}$ & 41.5 & 5.8 & -1.2 & -8.3 \\
\hline $\mathrm{Zr}$ & 48.2 & 9.8 & -143 & -148 \\
\hline $\mathrm{Cr}$ & 31.5 & -70 & -1.5 & -10.3 \\
\hline $\mathrm{Ti}$ & 22.8 & 16.3 & -77.2 & -95.4 \\
\hline $\mathrm{Nb}$ & 0 & 0 & -10.3 & -90.6 \\
\hline
\end{tabular}




\section{List of Figure Captions}

Figure 1. Schematic illustration of $\Delta G_{\text {amorph }}$ in a hypothetic ternary $A-B-C$ system.

Figure 2. Schematic illustration of the two-step procedure to construct an isothermal section of a ternary GB $\lambda$-diagram for $\underline{\mathrm{W}}-\mathrm{Ni}$-Fe at $1673 \mathrm{~K}$. (a) First, $\lambda\left(\mathbf{X}_{\text {bulk }}^{B C C}\right)$ values are computed and plotted at a function of bulk composition of the $\mathrm{BCC}$ phase without considering the precipitation of any secondary crystalline phase. (b) Subsequently, the precipitation of other equilibrium secondary phases (FCC and $\mu-\mathrm{FeW}$ in this specific case), which limit the bulk chemical potentials and $\lambda$ values in two- and three-phase coexistence regions, are considered. The corresponding binary GB $\lambda$ diagrams for (c) $\underline{\mathrm{W}}-\mathrm{Fe}$ and (d) $\underline{\mathrm{W}}-\mathrm{Ni}$. (e) A HRTEM image showing a nanoscale quasi-liquid IGF, adapted from Ref. [15] with permission.

Figure 3. (a) An uncolored computed GB $\lambda$ diagram for the average general GBs in $\underline{\mathrm{W}}-\mathrm{Ni}$-Fe at 1673 $\mathrm{K}$ and (b) the corresponding estimated GB-to-GB variations. Panel (a) shows four lines of constant $\lambda$ ( $\lambda=0.5,1,2$ and $4 \mathrm{~nm}$ ), while panel (b) only displays two bands that correspond to $\lambda=0.5$ and $2 \mathrm{~nm}$, respectively, for clarity.

Figure 4. Computed GB $\lambda$ diagrams suggesting that adding an alloying element $X$ that has a negative (positive) pair-interaction parameter with Mo can inhibit (promote) GB disorder. The HRTEM image is adapted from Ref. [19] with permission.

Figure 5. Computed GB $\lambda$ diagrams for $\underline{\mathrm{W}}-\mathrm{Ni}-X$ systems at $1673 \mathrm{~K}$ showing that the melting temperature of $X\left(T_{X}^{m}\right)$ has little impact on GB disordering in the $\mathrm{W}$ rich region. In this set of numerical experiments, $X$ was assumed to form ideal solutions with $\mathrm{W}$ and Ni for simplicity.

Figure 6. The effects of varying (a) $\Omega_{\mathrm{W}-X}^{\mathrm{BCC}}$, (b) $\Omega_{\mathrm{W}-X}^{\mathrm{L}}$, (c) $\Omega_{\mathrm{N}-X}^{\mathrm{BCC}}$ and (d) $\Omega_{\mathrm{Ni}-X}^{\mathrm{L}}$ on the computed GB $\lambda$ diagrams for $\underline{\mathrm{W}}-\mathrm{Ni}-X$ systems at $1673 \mathrm{~K}$. These calculations assumed that $T_{X}^{m}=2000 \mathrm{~K}$ and $X$ forms ideal solutions with $\mathrm{W}$ and $\mathrm{Ni}$ other than one regular solution, for which the $\Omega$ value is labeled below the diagram. 
Figure 7. Computed GB $\lambda$ diagrams for $\underline{\mathrm{W}}-\mathrm{Ni}-X$ systems at $1637 \mathrm{~K}$ with decreasing $\Omega_{\mathrm{W}-X}^{\mathrm{L}} / \Omega_{\mathrm{W}-X}^{\mathrm{BCC}}$ assuming that $\left(\Omega_{\mathrm{W}-X}^{\mathrm{BCC}}-\Omega_{\mathrm{W}-X}^{\mathrm{L}}\right)=+50 \mathrm{~kJ} / \mathrm{mol}, T_{X}^{m}=2000 \mathrm{~K}$ and $X$ forms ideal solutions with Ni.

Figure 8. (a) The density increases for three specimens (W, W-0.5Ni and $\mathrm{W}-0.5 \mathrm{Ni}-0.5 \mathrm{Fe}$ at. \%) after sintering at $1573 \mathrm{~K}$ for two hours $v s$. the computed $\lambda$ values for these three compositions. Adding 0.5 at. $\%$ $\mathrm{Ni}$ as a sintering aid significantly boosted the densification of $\mathrm{W}$ and adding 0.5 at. \% Fe as a co (the second) sintering aid further enhanced the densification. (b) The corresponding computed GB $\lambda$ diagram for $\underline{\mathrm{W}}-\mathrm{Ni}-\mathrm{Fe}$ at $1573 \mathrm{~K}$, in which the three selected composition points are labeled.

Figure 9. Computed GB $\lambda$ diagrams for the $\underline{\mathrm{W}}-\mathrm{Ni}-M(M=\mathrm{Cr}, \mathrm{Zr}, \mathrm{Co}, \mathrm{Fe}, \mathrm{Nb}$ and $\mathrm{Ti})$ systems at $1573 \mathrm{~K}$ considering (a) only the $\mathrm{BCC}$ and liquid phases (a.k.a. without considering the precipitation of secondary crystalline phases) and (b) all possible equilibrium phases, respectively. (c) Calculated $\lambda$ values generally correlate well with the measured density increases after 2-hour sintering at $1573 \mathrm{~K}$ for this series of ternary $\mathrm{W}-0.5 \mathrm{Ni}-0.5 \mathrm{M}$ (at. \%) alloys, along with binary $\mathrm{W}-0.5 \mathrm{Ni}$ and $\mathrm{W}-1 \mathrm{Ni}$ (at. \%) alloys as the references. Noting that the composition points of $\mathrm{W}-0.5 \mathrm{Ni}-0.5 \mathrm{M}$ (at. \%) are outside the plotted regions of these GB $\lambda$ diagrams (that are expanded to $0-0.3 \%$ Ni to clearly show the low concentration regions), but there are little changes in the computed $\lambda$ values beyond 0.3 at. $\% \mathrm{Ni}$.

Figure 10. (a) The relative effectiveness of adding 0.5 at. \% Ni, Co and $\mathrm{Fe}$ as sintering aids on the final sintered densities of Mo-Si-B alloys after isothermal sintering at $1873 \mathrm{~K}$ for 3 hours (repotted after the data in Ref. [17]). (b) A computed ternary GB $\lambda$ diagram for Mo-Si-B at 1873 K. (c, d, e) The pseudo-ternary sections of GB $\lambda$ diagrams for the quaternary $\underline{\mathrm{Mo}}-\mathrm{Si}$-B-0.5M systems at $1873 \mathrm{~K}$ and a fixed atomic fraction of 0.5 at. $\% M(M=\mathrm{Ni}, \mathrm{Co}$ and $\mathrm{Fe})(\mathbf{f}, \mathbf{g})$ Additional (new) experiments showed that adding 0.5 at. \% $\mathrm{Ni}$ (the most effective sintering aid predicted) substantially increased the densification of Mo-Si-B alloys to achieve $>97 \%$ of the theoretical density after 12 hours' sintering at $1873 \mathrm{~K}$. 


\section{References:}

[1] J.G. Dash, Surface Melting, Contemporary Physics 30 (1989) 89-100.

[2] J.G. Dash, H. Fu, J.S. Wettlaufer, The Premelting of Ice and Its Environmental Consequences, Reports on Progress in Physics 58 (1995) 115-167.

[3] J.G. Dash, A.M. Rempel, J.S. Wettlaufer, The Physics of Premelted Ice and Its Geophysical Consequences, Reviews of Modern Physics 78 (2006) 695-741.

[4] T.E. Hsieh, R.W. Balluffi, Experimental Study of Grain Boundary Melting in Aluminum, Acta Metall. 37 (1989) 1637-1644.

[5] A.M. Alsayed, M.F. Islam, J. Zhang, P.J. Collings, A.G. Yodh, Premelting at Defects within Bulk Colloidal Crystals, Science 309 (2005) 1207-1210.

[6] S. Divinski, M. Lohmann, C. Herzig, B. Straumal, B. Baretzky, W. Gust, Grain-boundary melting phase transition in the Cu-Bi system, Phys. Rev. B 71 (2005)

[7] B.B. Straumal, B. Baretzky, Grain Boundary Phase Transitions and their Influence on Properties of Ploycrystals, Interface Science 12 (2004) 147-155.

[8] L.-S. Chang, E.I. Rabkin, B. Straumal, P. Lejcek, S. Hofmann, W. Gust, Temperature Dependence of the Grain Boundary Segregation of Bi in Cu Polycrystals, Scr. Metall. Mater. 37 (1997) 729-735.

[9] E.I. Rabkin, V.N. Semenov, L.S. Shvindlerman, B.B. Straumal, Penetration of Tin and Zinc Along Tilt Grain-Boundaries 43-Degrees [100] in Fe-5 at-Percent Si Alloy - Premelting Phase-Transition, Acta Metall Mater 39 (1991) 627-639.

[10] O.I. Noskovich, E.I. Rabkin, V.N. Semenov, B.B. Straumal, L.S. Shvindlerman, Wetting and premelting phase transitions in $38^{\circ}$ [100] tilt grain boundary in (Fe-12 at.\% $\mathrm{Si}$ )- $\mathrm{Zn}$ alloy in the vicinity of the A2-B2 bulk ordering in Fe-12 at.\% Si alloy, Acta Metall Mater 39 (1991) 3091-3098.

[11] H. Wang, Y.-M. Chiang, Thermodynamic Stability of Intergranular Amorphous Films in BismuthDoped Zinc Oxide, J. Am. Ceram. Soc. 81 (1998) 89-96.

[12] J. Luo, H. Wang, Y.-M. Chiang, Origin of Solid State Activated Sintering in $\mathrm{Bi}_{2} \mathrm{O}_{3}$-Doped ZnO, J. Am. Ceram. Soc. 82 (1999) 916.

[13] I. MacLaren, R.M. Cannon, M.A. Gülgün, R. Voytovych, N.P. Pogrion, C. Scheu, U. Täffner, M. Rühle, Abnormal Grain Growth in Alumina: Synergistic Effects of Yttria and Silica, J. Am. Ceram. Soc. 86 (2003) 650.

[14] J. Luo, Stabilization of Nanoscale Quasi-Liquid Interfacial Films in Inorganic Materials: A Review and Critical Assessment, Critical Reviews in Solid State and Material Sciences 32 (2007) 67-109. 
[15] J. Luo, V.K. Gupta, D.H. Yoon, H.M. Meyer, Segregation-Induced Grain Boundary Premelting in Nickel-doped Tungsten, Appl. Phys. Lett. 87 (2005) 231902.

[16] V.K. Gupta, D.H. Yoon, H.M. Meyer III, J. Luo, Thin Intergranular Films and Solid-State Activated Sintering in Nickel-Doped Tungsten, Acta Mater. 55 (2007) 3131-3142.

[17] J.I. Jung, N.X. Zhou, J. Luo, Effects of sintering aids on the densification of Mo-Si-B alloys, J. Mater. Sci. 47 (2012) 8308-8319.

[18] X. Shi, J. Luo, Developing grain boundary diagrams as a materials science tool: A case study of nickel-doped molybdenum, Phys.Rev. B 84 (2011) 014105.

[19] X. Shi, J. Luo, Grain boundary wetting and prewetting in Ni-doped Mo, Appl. Phys. Lett. 94 (2009) 251908.

[20] M. Tang, W.C. Carter, R.M. Cannon, Grain Boundary Transitions in Binary Alloys, Phys. Rev. Lett. 97 (2006) 075502.

[21] Y. Mishin, W.J. Boettinger, J.A. Warren, G.B. McFadden, Thermodynamics of grain boundary premelting in alloys. I. Phase-field modeling, Acta Mater. 57 (2009) 3771-3785.

[22] J.W. Cahn, Critical Point Wetting, J. Chem. Phys. 66 (1977) 3667-3672.

[23] J. Monk, D. Farkas, Strain-induced grain growth and rotation in nickel nanowires, Phys. Rev. B 75 (2007) 045414.

[24] M.D. McMurtrey, G.S. Was, L. Patrick, D. Farkas, Relationship between localized strain and irradiation assisted stress corrosion cracking in an austenitic alloy, Mater.Sci. Eng. A 528 (2011) 3730-3740.

[25] M.P. Seah, Grain Boundary Segregation, Journal of Physics F: Metal Physics 10 (1980) 1043-1064.

[26] E.D. Hondros, M.P. Seah, The Theory of Grain Boundary Segregation in Terms of Surface Adsorption Analogues, Metall. Trans. 8A (1977) 1363-1371.

[27] J.W. Cahn, Transition and Phase Equilibria among Grain Boundary Structures, J. de Physique 43 (1982) C6.

[28] R. Kikuchi, J.W. Cahn, Grain Boundary Melting Transition in a Two-Dimensional Lattice-Gas Model, Phys. Rev. B 21 (1980) 1893-1897.

[29] R. Kikuchi, J.W. Cahn, Grain Boundaries with Impurities in a Two-Dimensional Lattice-Gas Model, Phys. Rev. B 36 (1987) 418

[30] D.R. Clarke, On the Equilibrium Thickness of Intergranular Glass Phases in Ceramic Materials, J. Am. Ceram. Soc. 70 (1987) 15-22. 
[31] D.R. Clarke, T.M. Shaw, A.P. Philipse, R.G. Horn, Possible Electrical Double-Layer Contribution to the Equilibrium Thickness of Intergranular Glass Films in Polycrystalline Ceramics, J. Am. Ceram. Soc. 76 (1993) 1201-1204.

[32] M. Tang, W.C. Carter, R.M. Cannon, Diffuse Interface Model for Structural Transitions of Grain Boundaries, Phys. Rev. B 73 (2006) 024102.

[33] C.M. Bishop, R.M. Cannon, W.C. Carter, A Diffuse Interface Model of Interfaces: Grain Boundaries in Silicon Nitride, Acta Mater. 53 (2005) 4755-4764.

[34] W.D. Kaplan, D. Chatain, P. Wynblatt, W.C. Carter, A review of wetting versus adsorption, complexions, and related phenomena: the rosetta stone of wetting J. Mater. Sci. 48 (2013) 56815717.

[35] M. Tang, W.C. Carter, R.M. Cannon, Grain Boundary Order-Disorder Transitions, J. Mater. Sci. 41 (2006) 7691-7695.

[36] C.M. Bishop, M. Tang, R.M. Cannon, W.C. Carter, Continuum Modelling and Representations of Interfaces and Their Transitions in Materials, Mater. Sci. Eng. A 422 (2006) 102-114.

[37] P. Wynblatt, D. Chatain, Solid-state wetting transitions at grain boundaries, Mater. Sci. Eng. A 495 (2008) 119-125.

[38] P. Wynblatt, D. Chatain, Anisotropy of segregation at grain boundaries and surfaces (vol 37A, pg 2595, 2006), Metall. Mater, Trans. 37A 38A (2007) 438-439.

[39] P. Wynblatt, D. Chatain, Anisotropy of segregation at grain boundaries and surfaces, Metall. Mater, Trans. 37A (2006) 2595-2620.

[40] J. Luo, Grain boundary complexions: The interplay of premelting, prewetting, and multilayer adsorption, Appl. Phys. Lett. 95 (2009) 071911.

[41] J. Luo, Developing Interfacial Phase Diagrams for Applications in Activated Sintering and Beyond: Current Status and Future Directions, J. Am. Ceram. Soc. 95 (2012) 2358-2371.

[42] J. Luo, X.M. Shi, Grain boundary disordering in binary alloys, Appl. Phys. Lett. 92 (2008 ) 101901

[43] R.H. French, V.A. Parsegian, R. Podgornik, R.F. Rajter, A. Jagota, J. Luo, D. Asthagiri, M.K. Chaudhury, Y.M. Chiang, S. Granick, S. Kalinin, M. Kardar, R. Kjellander, D.C. Langreth, J. Lewis, S. Lustig, D. Wesolowski, J.S. Wettlaufer, W.Y. Ching, M. Finnis, F. Houlihan, O.A. von Lilienfeld, C.J. van Oss, T. Zemb, Long range interactions in nanoscale science, Reviews of Modern Physics 82 (2010) 1887-1944.

[44] R.H. French, Origin and Applications of London Dispersion Forces and Hamaker Constants in Ceramics, J. Am. Ceram. Soc. 83 (2000) 2117-2146 
[45] R.M. Cannon, M. Rühle, M.J. Hoffmann, R.H. French, H. Gu, A.P. Tomsia, E. Saiz, Adsorption and Wetting Mechanisms at Ceramic Grain Boundaries, Ceramic Transactions (Grain Boundary Engineering in Ceramics) 118 (2000) 427-444.

[46] P.R. Cantwell, M. Tang, S.J. Dillon, J. Luo, G.S. Rohrer, M.P. Harmer, Overview No. 152: Grain boundary complexions, Acta Mater. 62 (2014) 1-48.

[47] S.J. Dillon, M.P. Harmer, Multiple Grain Boundary Transitions in Ceramics: A Case Study of Alumnia, Acta Mater. 55 (2007) 5247-5254.

[48] S.J. Dillon, M. Tang, W.C. Carter, M.P. Harmer, Complexion: A new concept for kinetic engineering in materials science, Acta Mater. 55 (2007) 6208-6218

[49] M.P. Harmer, Interfacial Kinetic Engineering: How Far Have We Come Since Kingery's Inaugural Sosman Address?, J. Am. Ceram. Soc. 93 (2010) 301-317.

[50] M.P. Harmer, The Phase Behavior of Interfaces, Science 332 (2011) 182-183.

[51] S. Ma, K. Meshinchi Asl, C. Tansarawiput, P.R. Cantwella, M. Qi, M.P. Harmer, J. Luo, A GrainBoundary Phase Transition in Si-Au, Scr. Mater. 66 (2012) 203-206.

[52] A. Kundu, K.M. Asl, J. Luo, M.P. Harmer, Identification of a Bilayer Grain Boundary Complexion in Bi-doped Cu, Scr. Mater. 68 (2012) 146-149.

[53] J. Luo, H. Cheng, K.M. Asl, C.J. Kiely, M.P. Harmer, The Role of a Bilayer Interfacial Phase on Liquid Metal Embrittlement, Science 333 (2011) 1730-1733.

[54] X. Shi, J. Luo, Decreasing the Grain Boundary Diffusivity in Binary Alloys with Increasing Temperature, Phys. Rev. Lett. 105 (2010) 236102.

[55] S.J. Dillon, M.P. Harmer, J. Luo, Grain Boundary Complexions in Ceramics and Metals: An Overview, JOM 61 [12] (2009) 38-41.

[56] S. Ma, P.R. Cantwell, T.J. Pennycook, N. Zhou, M.P. Oxley, D.N. Leonard, S.J. Pennycook, J. Luo, M.P. Harmer, Grain boundary complexion transitions in $\mathrm{WO}_{3}$ - and $\mathrm{CuO}$-doped $\mathrm{TiO}_{2}$ bicrystals, Acta Mater. 61 (2013) 1691-1704.

[57] T. Frolov, D.L. Olmsted, M. Asta, Y. Mishin, Structural phase transformations in metallic grain boundaries, Nature Communications 4 (2013) 1899.

[58] T. Frolov, S.V. Divinski, M. Asta, Y. Mishin, Effect of Interface Phase Transformations on Diffusion and Segregation in High-Angle Grain Boundaries, Phys. Rev. Lett. 110 (2013) 255502.

[59] H. Qian, J. Luo, Nanoscale Surficial Films and A Surface Transition in V2O5-TiO2-Based Ternary Oxide Systems, Acta Mater. 56 (2008) 4702-4714. 
[60] J. Luo, Y.-M. Chiang, Wetting and Prewetting on Ceramic Surfaces, Annu. Rev. Mater. Res. 38 (2008) 227-249.

[61] H.J. Qian, J. Luo, Vanadia-Based Equilibrium-Thickness Amorphous Films on Anatase (101) Surfaces, Appl. Phys. Lett. 91 (2007) 061909.

[62] J. Luo, Y.-M. Chiang, R.M. Cannon, Nanometer-Thick Surficial Films in Oxides as A Case of Prewetting, Langmuir 21 (2005) 7358-7365.

[63] S.J. Dillon, M.P. Harmer, Demystifying the role of sintering additives with "complexion", J. Eur. Ceram. Soc. 28 (2008) 1485-1493.

[64] J. Mellenthin, A. Karma, M. Plapp, Phase-field crystal study of grain-boundary premelting, Physical Review B 78 (2008) 184110.

[65] Y.-L. Lu, T.-T. Hu, G.-M. Lu, Z. Chen, Phase-field crystal study of segregation induced grainboundary premelting in binary alloys, Physica B: Condensed Matter 451 (2014) 128-133.

[66] J. Luo, Liquid-like interface complexion: From activated sintering to grain boundary diagrams, Current Opinion Solid State and Materials Science 12 (2008) 81-88.

[67] V.K. Gupta, D.H. Yoon, J. Luo, H.M. Meyer III, Preliminary Results of Activated Sintering Mechanism and Grain Boundary Prewetting/ Premelting in Nickel-doped Tungsten. In: R. M. Lane MZH, S. Lu, editor. Ceramic Nanomaterials and Nanotechnology IV, vol. 172: The American Ceramic Society, 2005. p.159-174.

[68] J.S. Lee, K. Klockgeter, C. Herzig, Grain Boundary Self and Impurity Diffusion in Tungsten in the Tempearture Range of Activated Sintering, Colloque De Physique 51 (1990) C1-569.

[69] S. Dietrich, Wetting Phenomena. In: Domb C, Lebowitz JL, editors. Phase Transitions and Critical Phenomena, vol. 12. London: Academic Press, 1988. p.1-218.

[70] J.J. Hoyt, D. Olmsted, S. Jindal, M. Asta, A. Karma, Method for computing short-range forces between solid-liquid interfaces driving grain boundary premelting, Phys. Rev. E 79 (2009) 020601.

[71] L.P.H. Jeurgens, Z. Wang, E.J. Mittemeijer, Thermodynamics of reactions and phase transformations at interfaces and surfaces, Int. J. Mater. Res. 100 (2009) 1281-1307.

[72] R. Benedictus, A. Böttger, E.J. Mittemeijer, Thermodynamic model for solid-state amorphization in binary systems at interfaces and grain boundaries, Phys. Rev. B 54 (1996) 9109-9125.

[73] N. Eustathopoulos, M. Nicholas, B. Drevet, Wettabilty At High Temperatures Amsterdam: Pergamon, 1999.

[74] H. Bakker, Enthalpies in Alloys: Miedema's Semi-Empirical Model: Enfield Publishing \& Distribution Company, 1998. 
[75] A.F. Guillermet, An Assessment of the Fe-Ni-W-C Phase Diagram, Z. Metallkde 78 (1987) 165171.

[76] P. Gustafson, A Thermodynamic Evaluation of the Cr-Ni-W System, CALPHAD: Comput. Coupling Phase Diagrams Thermochem. 12 (1988) 277-292.

[77] K. Frisk, A thermodynamic evaluation of the Mo-Ni system, CALPHAD: Comput. Coupling Phase Diagrams Thermochem. 14 (1990) 311-320.

[78] E.N. Hodkin, M.G. Nicholas, D.M. Poole, The surface energies of solid molybdenum, niobium, tantalum and tungsten, J. Less Common Met. 20 (1970) 93-103.

[79] P. Gustafson, A Thermodynamic Evaluation of the C-Fe-W System, Metall. Trans. A 18 (1987) 175-188.

[80] A. Guillermet, Thermodynamic properties of the Co-W-C system, Metall. Trans. A 20 (1989) 935956.

[81] A.F. Guillermet, Assessment of the thermodynamic properties of the Ni-Co System, Z. Metallkde 78 (1987) 639-647.

[82] A. Bolcavage, U.R. Kattner, A Reassessment of the Calculated Ni-Nb Phase Diagram, Phase Equilibria 17 (1996) 92-100.

[83] S.K. Lee, D.N. Lee, Calculation of phase diagrams using partial phase diagram data, CALPHAD: Comput. Coupling Phase Diagrams Thermochem 10 (1986) 61.

[84] N. Wang, C. Li, Z. Du, F. Wang, Experimental study and thermodynamic re-assessment of the NiZr system, CALPHAD: Comput. Coupling Phase Diagrams Thermochem 31 (2007) 413-421.

[85] S. Jonsson, Reevaluation of the Ti-W System and Prediction of the Ti-W-N Phase Diagram, Z. Metallkde 87 (1996) 784.

[86] P. Bellen, K.C. Hari Kumar, P. Wollants, Thermodynamic Assessment of the Ni-Ti Phase Diagram, Z. Metallkde 87 (1996) 972-978.

[87] J.K. Cochran, W.L. Daloz, P.E. Marshall, Oxidation resistant Mo-Mo(2)B-silica and Mo-Mo(2)Bsilicate composites for high temperature applications, JOM 63 [12] (2011) 44-49.

[88] Y. Yang, Y.A. Chang, Thermodynamic modeling of the Mo-Si-B system, Intermetallics 13 (2005) 121-128.

[89] Y. Liu, G. Shao, P. Tsakiropoulos, Thermodynamic Reassessment of the Mo-Si and Al-Mo-Si Systems, Intermetallics 8 (2000) 953-962.

[90] K.E. Spear, M.S. Wang, Thermodynamic modeling of the molybdenum-boron system, CALPHAD: : Comput. Coupling Phase Diagrams Thermochem. 5 (1981) 109-113. 
[91] M. Heilmaier, M. Kruger, H. Saage, J. Rosler, D. Mukherji, U. Glatzel, R. Volkl, R. Huttner, G. Eggeler, C. Somsen, T. Depka, H.J. Christ, B. Gorr, S. Burk, Metallic materials for structural applications beyond nickel-based superalloys, JOM 61 [7] (2009) 61-67.

[92] L. Kaufman, Coupled phase diagrams and thermochemical data for transition metal binary systemsVI, Calphad 3 (1979) 45-76.

[93] O. Teppo, P. Taskinen, Thermodynamic Assessment of Ni-B Phase-Diagram, Mater. Sci. \& Techn. 9 (1993) $205-212$.

[94] A. Davydov, U.R. Kattner, Thermodynamic assessment of the Co-Mo system, J. Phase Equilib. 20 (1999) 5-16.

[95] C. Soon-Don, Thermodynamic analysis of the Co-Si system, Calphad 16 (1992) 151-159.

[96] M. Hillert, C. Qiu, A Reassessment of the Fe-Cr-Mo-C System, J. Phase Equilibra 13 (1992) $512-$ 521.

[97] B. Hallemans, P. Wollants, J.R. Roos, Thermodynamic Reassessment and Calculation of the Fe-B Diagram, Z. Metallkd. 85 (1994) 676-682.

[98] J. Lacaze, B. Sundman, An assessment of the Fe-C-Si system, Metall. Trans. A 22 (1991) 22112223. 


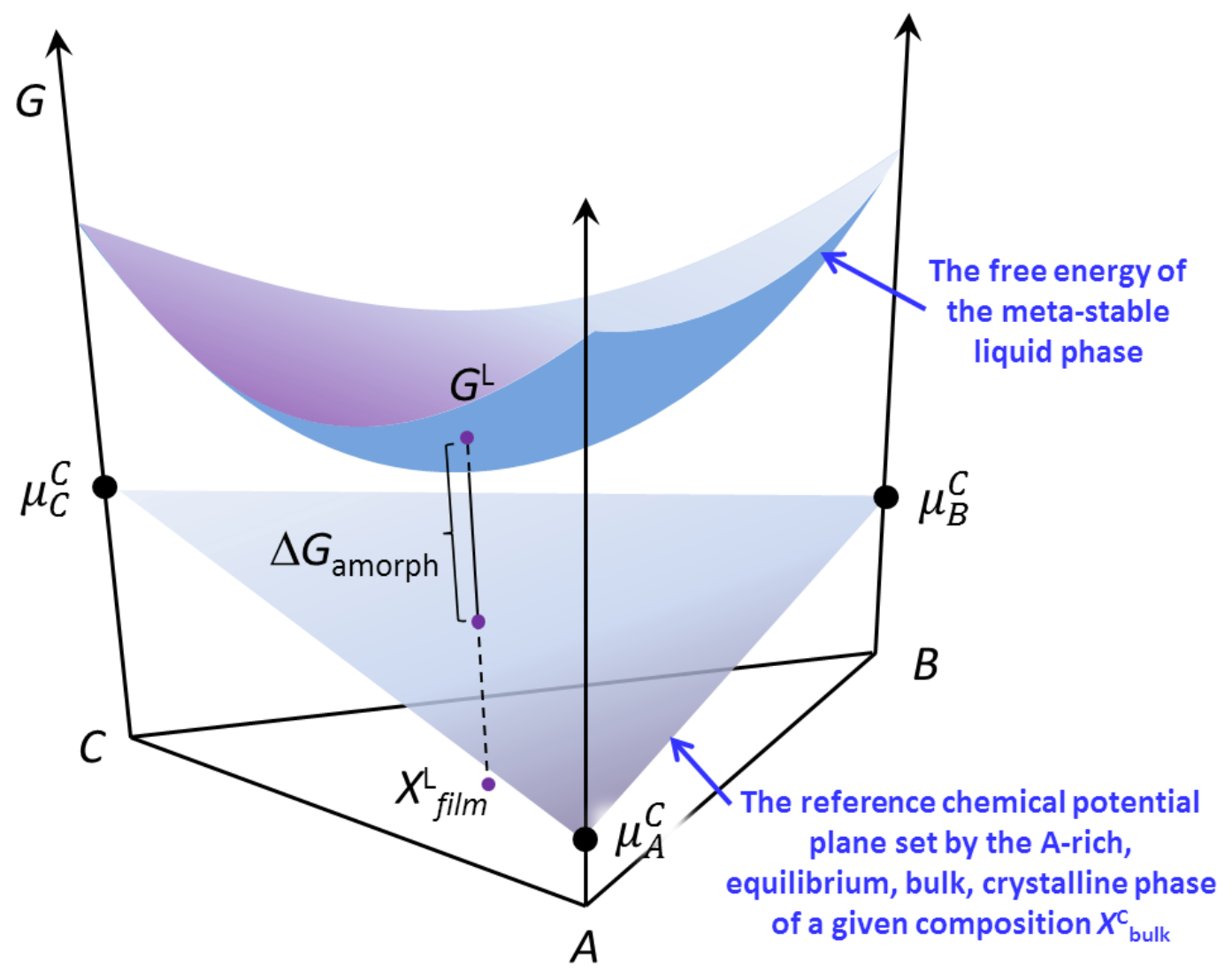

Figure 1. Schematic illustration of $\Delta G_{\text {amorph }}$ in a hypothetic ternary $A-B-C$ system. 


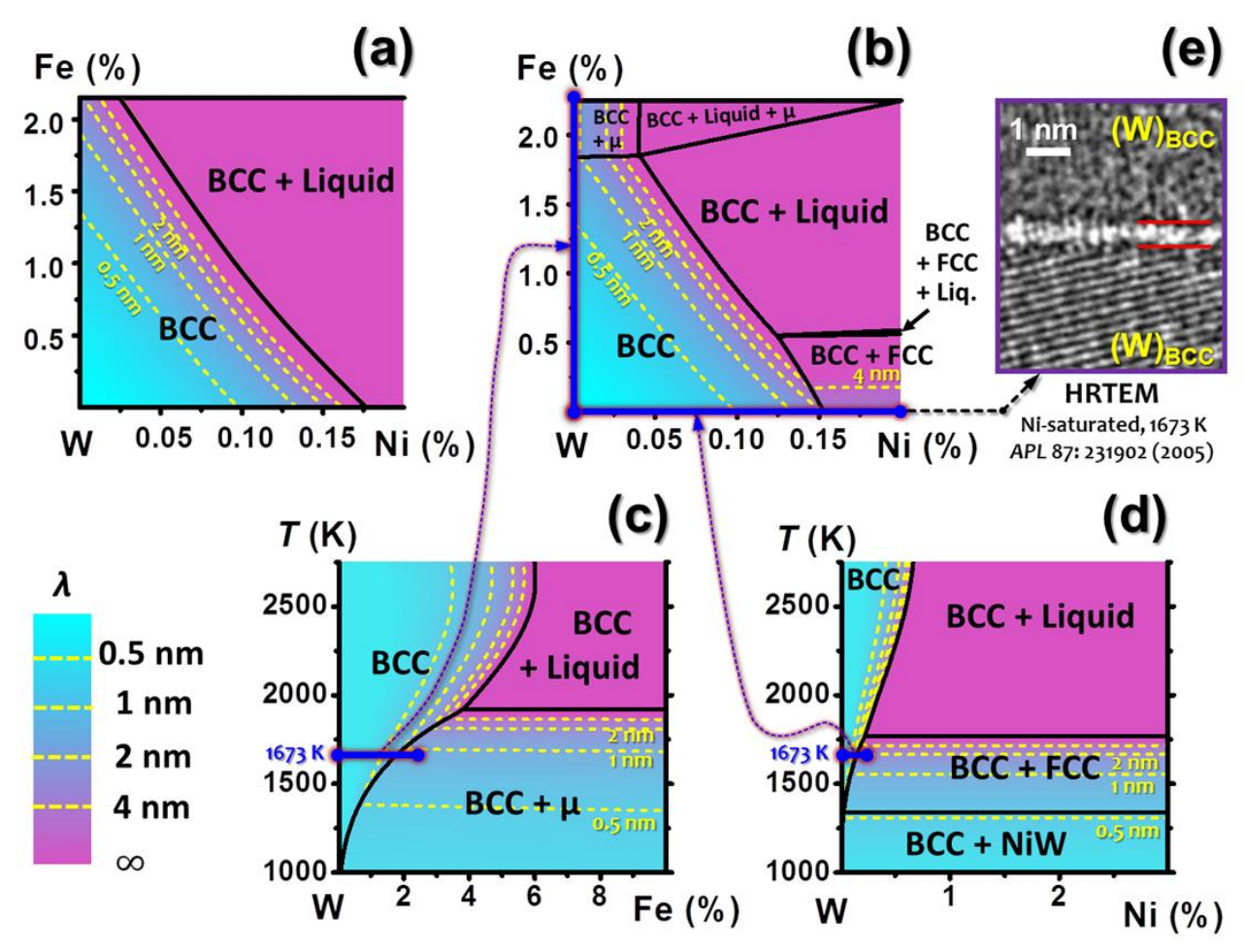

Figure 2. Schematic illustration of the two-step procedure to construct an isothermal section of a ternary GB $\lambda$-diagram for $\underline{\mathrm{W}}-\mathrm{Ni}$-Fe at $1673 \mathrm{~K}$. (a) First, $\lambda\left(\mathbf{X}_{\text {bulk }}^{B C C}\right)$ values are computed and plotted at a function of bulk composition of the $\mathrm{BCC}$ phase without considering the precipitation of any secondary crystalline phase. (b) Subsequently, the precipitation of other equilibrium secondary phases (FCC and $\mu$-FeW in this specific case), which limit the bulk chemical potentials and $\lambda$ values in two- and three-phase coexistence regions, are considered. The corresponding binary GB $\lambda$ diagrams for (c) $\underline{\mathrm{W}}-\mathrm{Fe}$ and (d) $\underline{\mathrm{W}}-\mathrm{Ni}$. (e) A HRTEM image showing a nanoscale quasi-liquid IGF, adapted from Ref. [15] with permission. 

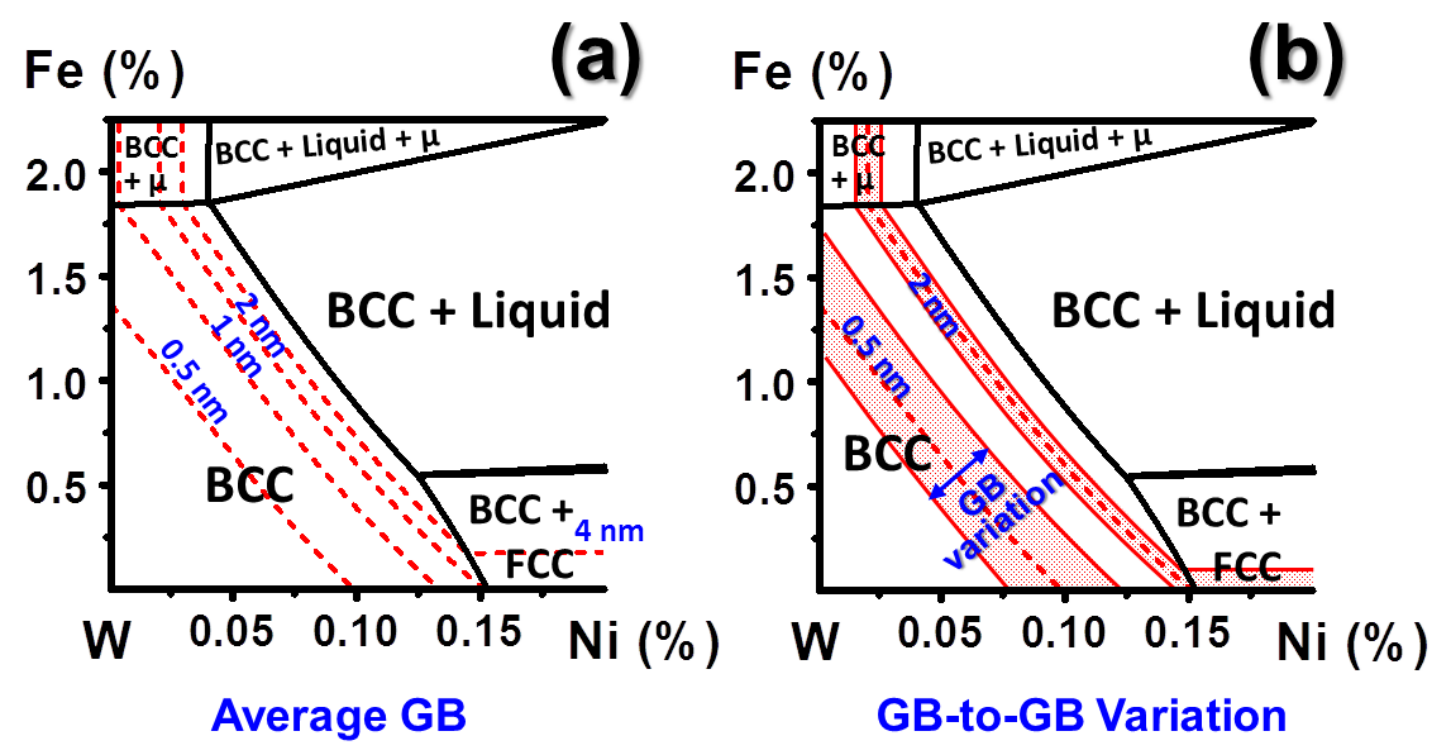

Figure 3. (a) An uncolored computed GB $\lambda$ diagram for the average general GBs in $\underline{\mathrm{W}}-\mathrm{Ni}-\mathrm{Fe}$ at 1673 $\mathrm{K}$ and (b) the corresponding estimated GB-to-GB variations. Panel (a) shows four lines of constant $\lambda$ $(\lambda=0.5,1,2$ and $4 \mathrm{~nm}$ ), while panel (b) only displays two bands that correspond to $\lambda=0.5$ and $2 \mathrm{~nm}$, respectively, for clarity. 


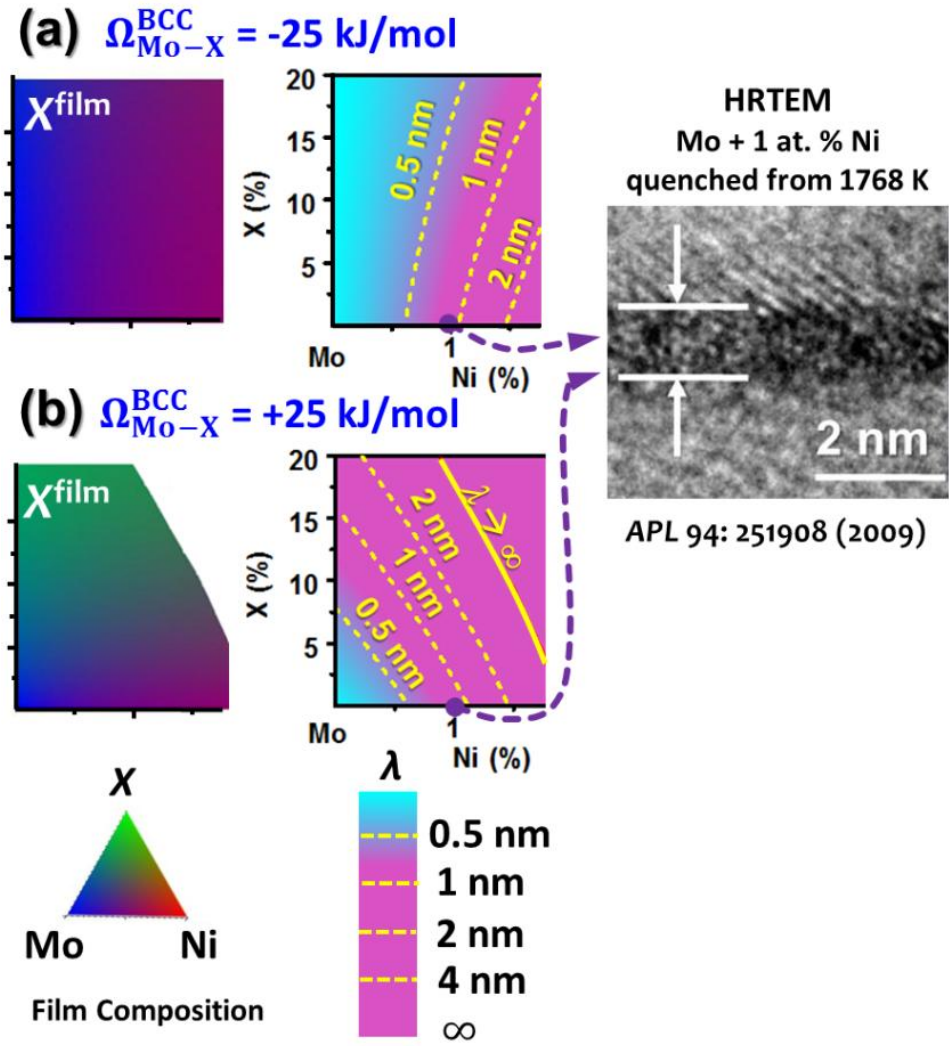

Figure 4. Computed GB $\lambda$ diagrams suggesting that adding an alloying element $X$ that has a negative (positive) pair-interaction parameter with Mo can inhibit (promote) GB disorder. The HRTEM image is adapted from Ref. [19] with permission. 

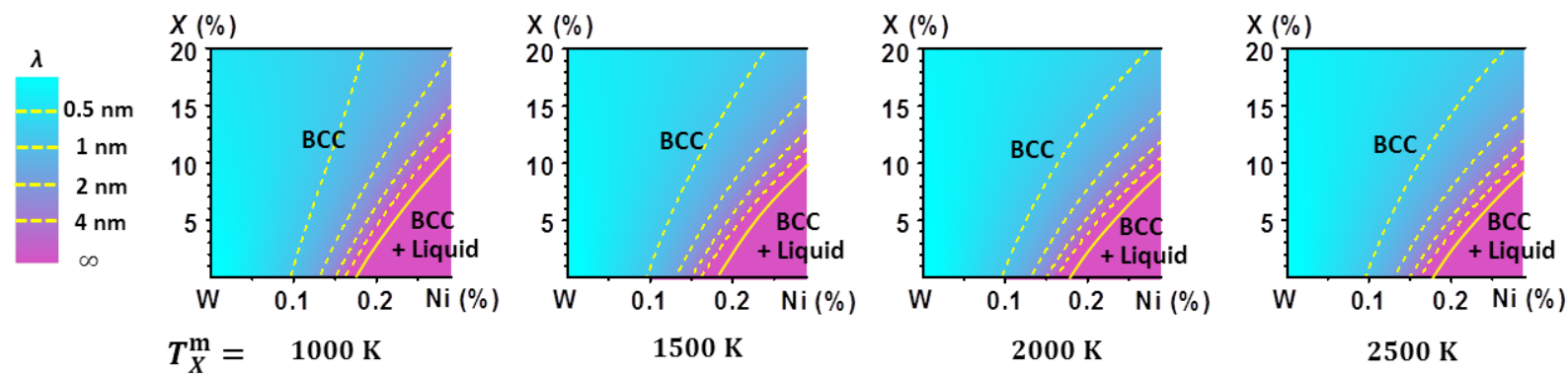

Figure 5. Computed GB $\lambda$ diagrams for $\underline{\mathrm{W}}-\mathrm{Ni}-X$ systems at $1673 \mathrm{~K}$ showing that the melting temperature of $X\left(T_{X}^{m}\right)$ has little impact on GB disordering in the $\mathrm{W}$ rich region. In this set of numerical experiments, $X$ was assumed to form ideal solutions with $\mathrm{W}$ and Ni for simplicity. 
(a)

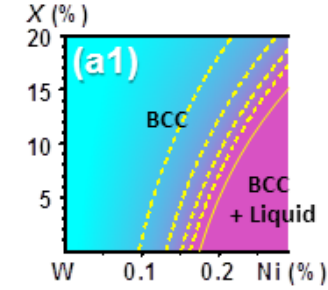

$\Omega_{X-W}^{\mathrm{BCC}}=-50 \mathrm{~kJ} / \mathrm{mol}$
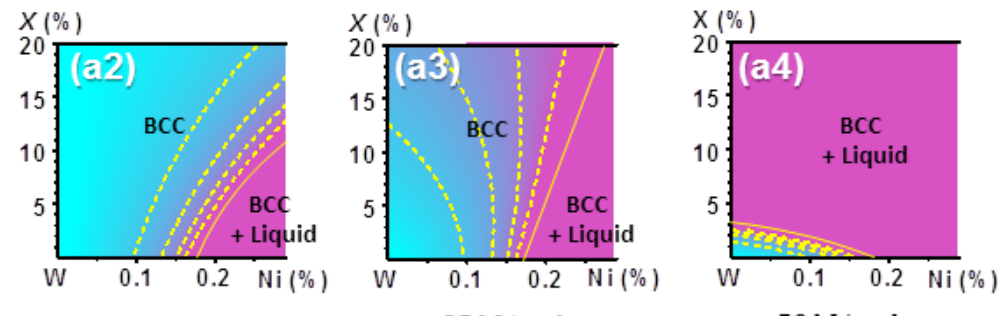

(b)

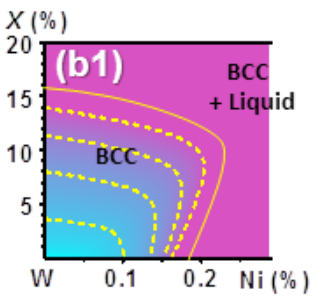

$\Omega_{X-W}^{\mathrm{L}}=\quad-75 \mathrm{~kJ} / \mathrm{mol}$

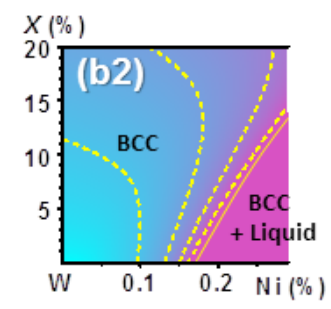

$-50 \mathrm{~kJ} / \mathrm{mol}$

$25 \mathrm{~kJ} / \mathrm{mol}$

$50 \mathrm{~kJ} / \mathrm{mol}$
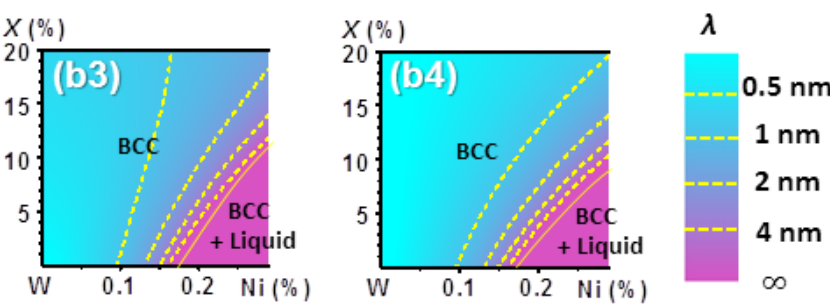

(c)

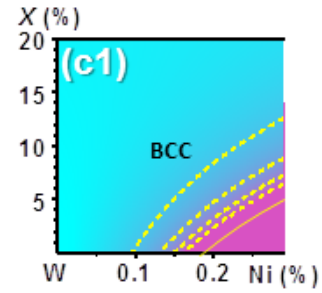

$\Omega_{X-\mathrm{Ni}}^{\mathrm{BCC}}=-50 \mathrm{~kJ} / \mathrm{mol}$

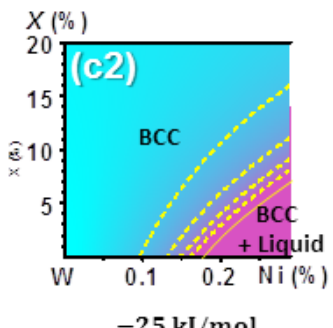

$-25 \mathrm{~kJ} / \mathrm{mol}$

$25 \mathrm{~kJ} / \mathrm{mol}$
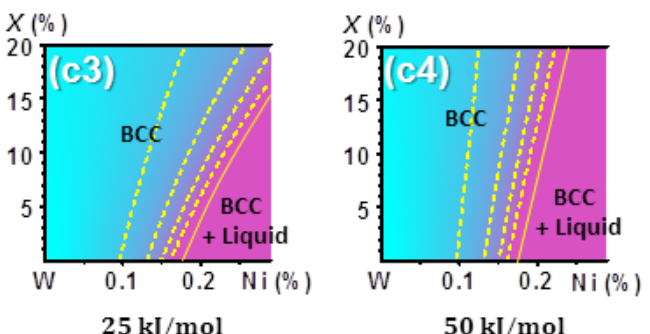

(d)

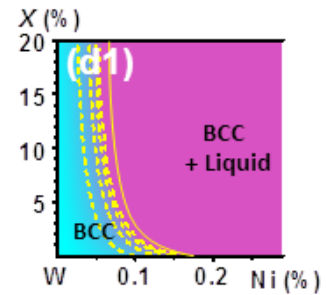

$\Omega_{X-\mathrm{Ni}}^{\mathrm{L}}=-75 \mathrm{~kJ} / \mathrm{mol}$
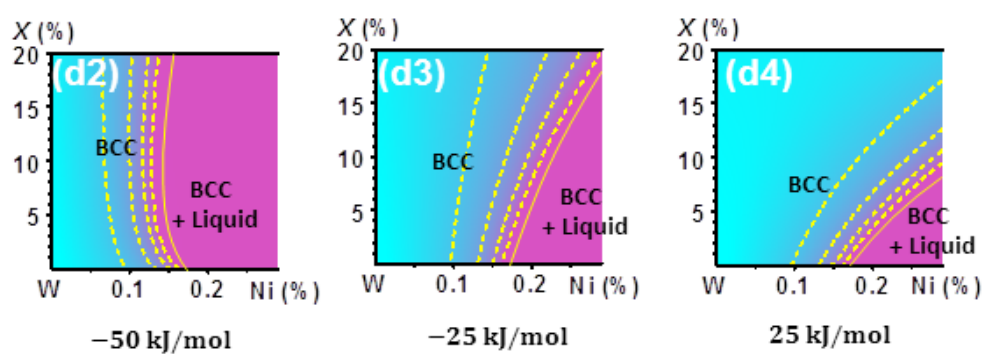

Figure 6. The effects of varying (a) $\Omega_{\mathrm{w}-X}^{\mathrm{BCC}}$, (b) $\Omega_{\mathrm{W}-X}^{\mathrm{L}}$, (c) $\Omega_{\mathrm{N}-\mathrm{X}}^{\mathrm{BCC}}$ and (d) $\Omega_{\mathrm{N}-\mathrm{X}}^{\mathrm{L}}$ on the computed GB $\lambda$ diagrams for $\underline{\mathrm{W}}-\mathrm{Ni}-X$ systems at $1673 \mathrm{~K}$. These calculations assumed that $T_{X}^{m}=2000 \mathrm{~K}$ and $X$ forms ideal solutions with $\mathrm{W}$ and $\mathrm{Ni}$ other than one regular solution, for which the $\Omega$ value is labeled below the diagram. 

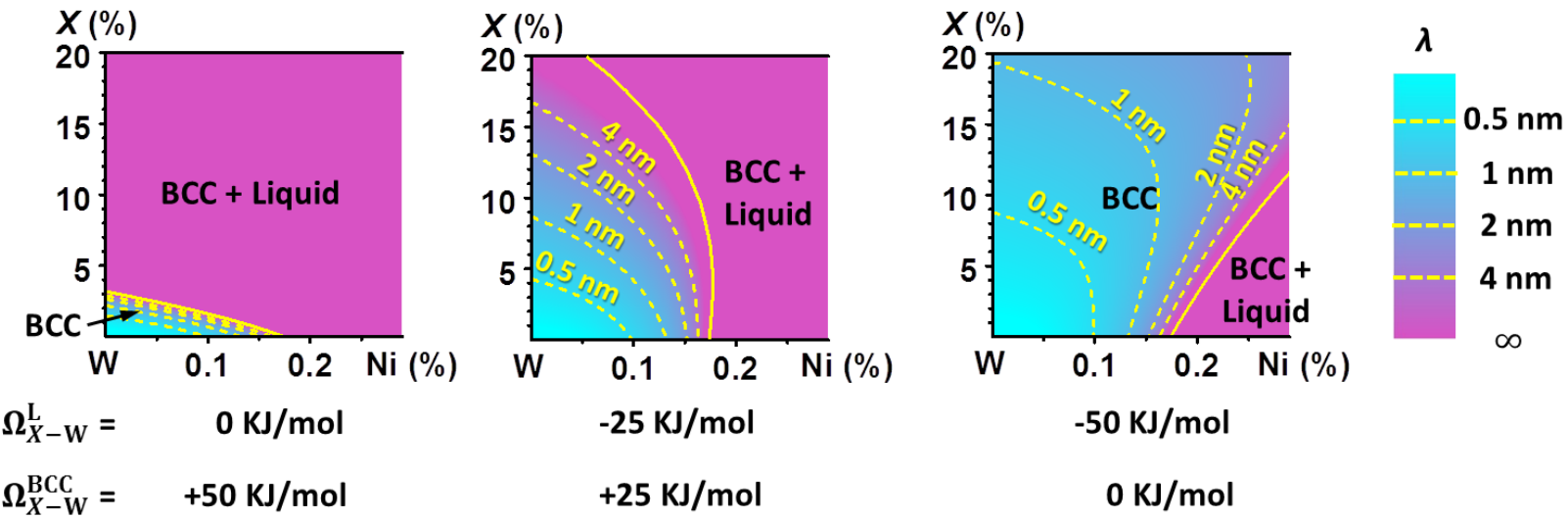

Figure 7. Computed GB $\lambda$ diagrams for $\underline{\mathrm{W}}-\mathrm{Ni}-X$ systems at $1637 \mathrm{~K}$ with decreasing $\Omega_{\mathrm{W}-X}^{\mathrm{L}} / \Omega_{\mathrm{W}-X}^{\mathrm{BCC}}$ assuming that $\left(\Omega_{\mathrm{W}-X}^{\mathrm{BCC}}-\Omega_{\mathrm{W}-X}^{\mathrm{L}}\right)=+50 \mathrm{~kJ} / \mathrm{mol}, T_{X}^{m}=2000 \mathrm{~K}$ and $X$ forms ideal solutions with Ni. 

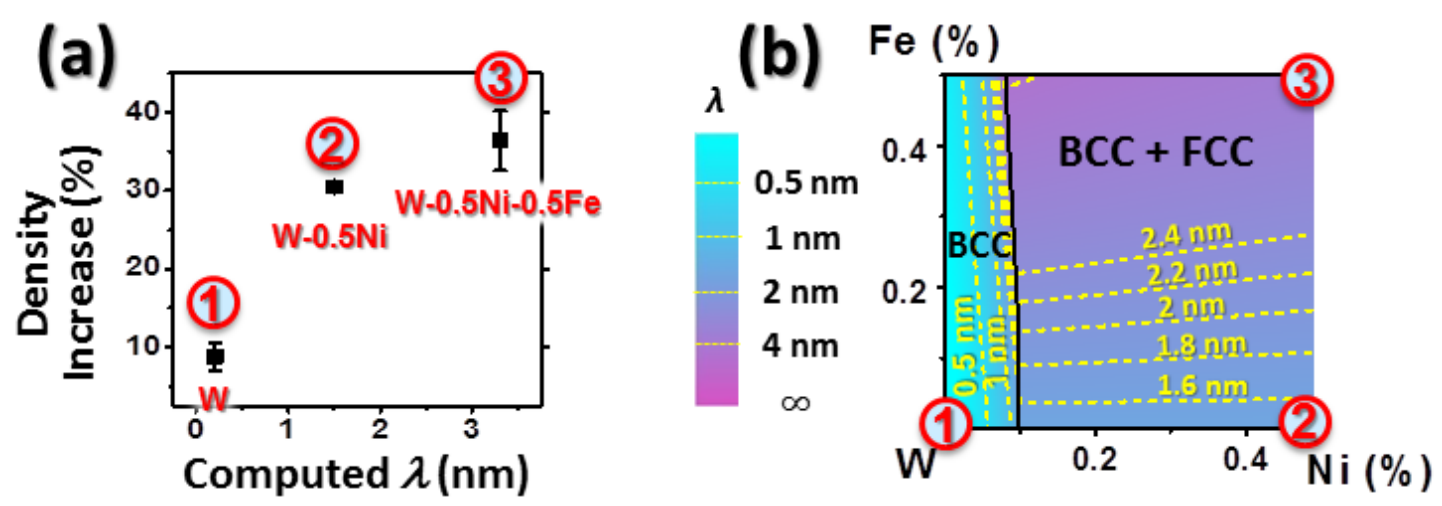

Figure 8. (a) The density increases for three specimens (W, W- $-0.5 \mathrm{Ni}$ and $\mathrm{W}-0.5 \mathrm{Ni}-0.5 \mathrm{Fe}$ at. \%) after sintering at $1573 \mathrm{~K}$ for two hours $v s$. the computed $\lambda$ values for these three compositions. Adding 0.5 at. $\%$ $\mathrm{Ni}$ as a sintering aid significantly boosted the densification of $\mathrm{W}$ and adding 0.5 at. $\% \mathrm{Fe}$ as a co (the second) sintering aid further enhanced the densification. (b) The corresponding computed GB $\lambda$ diagram for $\underline{\mathrm{W}}-\mathrm{Ni}-\mathrm{Fe}$ at $1573 \mathrm{~K}$, in which the three selected composition points are labeled. 

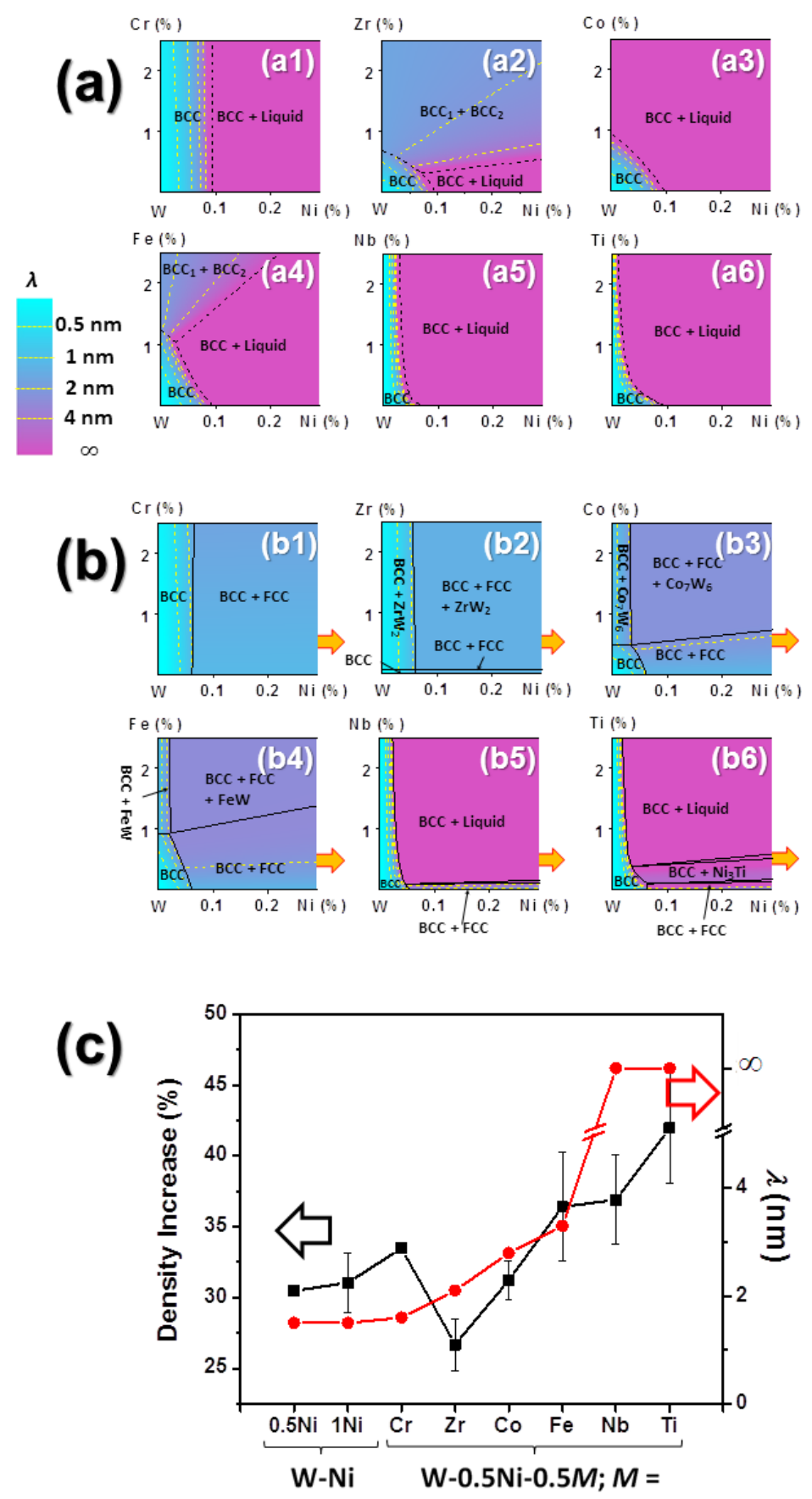

Figure 9. Computed GB $\lambda$ diagrams for the $\underline{\mathrm{W}}-\mathrm{Ni}-M(M=\mathrm{Cr}, \mathrm{Zr}, \mathrm{Co}, \mathrm{Fe}, \mathrm{Nb}$ and $\mathrm{Ti})$ systems at $1573 \mathrm{~K}$ considering (a) only the BCC and liquid phases (a.k.a. without considering the precipitation of secondary crystalline phases) and (b) all possible equilibrium phases, respectively. (c) Calculated $\lambda$ values generally correlate well with the measured density increases after 2-hour sintering at $1573 \mathrm{~K}$ for this series of ternary $\mathrm{W}-0.5 \mathrm{Ni}-0.5 \mathrm{M}$ (at. \%) alloys, along with binary $\mathrm{W}-0.5 \mathrm{Ni}$ and $\mathrm{W}-1 \mathrm{Ni}$ (at. \%) alloys as the references. Noting that the composition points of $\mathrm{W}-0.5 \mathrm{Ni}-0.5 \mathrm{M}$ (at. \%) are outside the plotted regions of these GB $\lambda$ diagrams (that are expanded to $0-0.3 \%$ Ni to clearly show the low concentration regions), but there are little changes in the computed $\lambda$ values beyond 0.3 at. $\% \mathrm{Ni}$. 

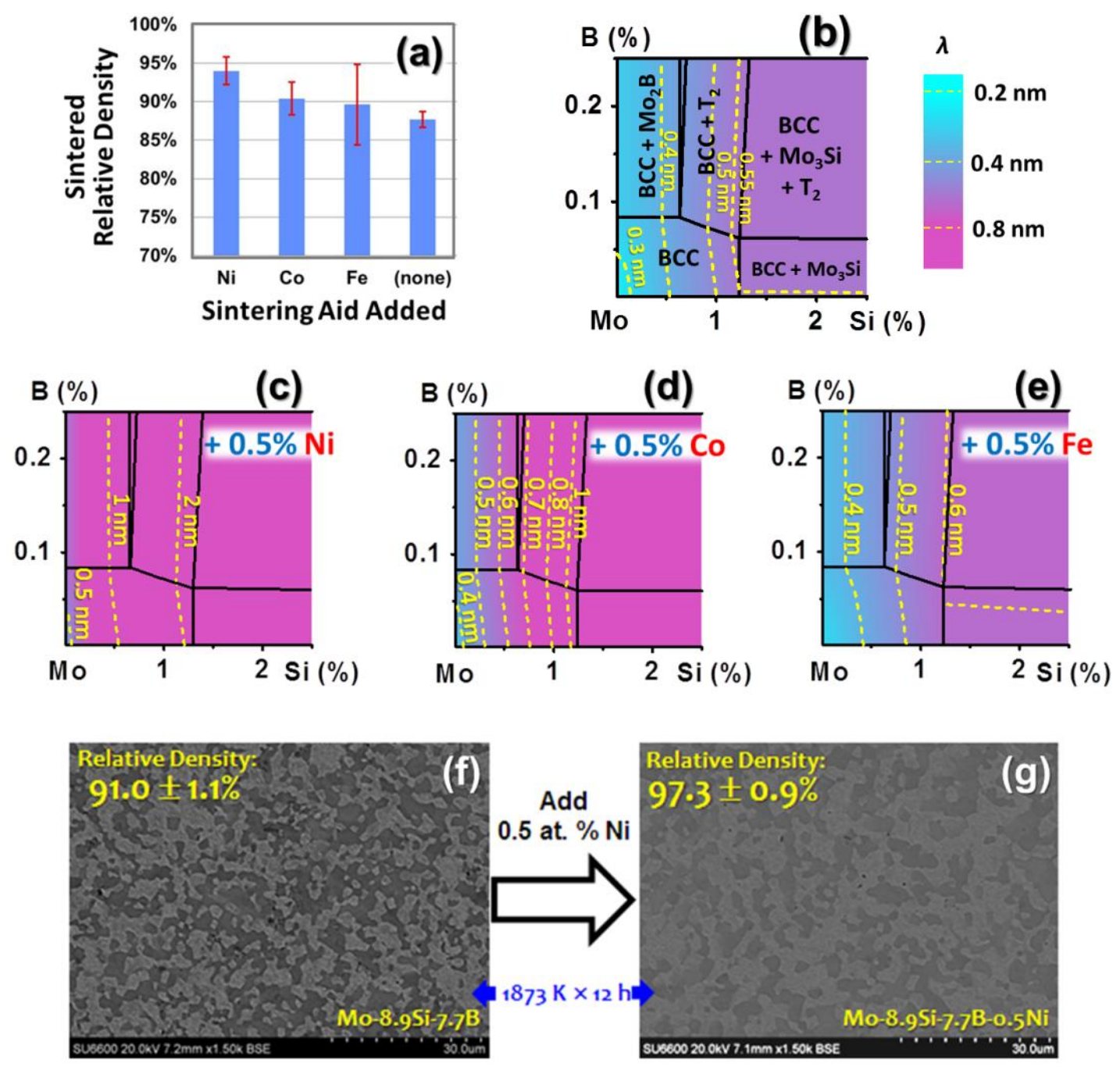

Figure 10. (a) The relative effectiveness of adding 0.5 at. $\% \mathrm{Ni}$, $\mathrm{Co}$ and $\mathrm{Fe}$ as sintering aids on the final sintered densities of Mo-Si-B alloys after isothermal sintering at $1873 \mathrm{~K}$ for 3 hours (repotted after the data in Ref. [17]). (b) A computed ternary GB $\lambda$ diagram for Mo-Si-B at 1873 K. (c, d, e) The pseudo-ternary sections of GB $\lambda$ diagrams for the quaternary Mo-Si-B-0.5M systems at $1873 \mathrm{~K}$ and a fixed atomic fraction of 0.5 at. $\% M(M=\mathrm{Ni}, \mathrm{Co}$ and $\mathrm{Fe})(\mathbf{f}, \mathbf{g})$ Additional (new) experiments showed that adding 0.5 at. $\% \mathrm{Ni}$ (the most effective sintering aid predicted) substantially increased the densification of Mo-Si-B alloys to achieve $>97 \%$ of the theoretical density after 12 hours' sintering at $1873 \mathrm{~K}$. 